Research Paper

\title{
Assessment of Groundwater Quality in the Jajrood River Basin, Tehran, Iran: A Coupled Physicochemical and Hydrogeochemical Study
}

\author{
Maryam Givi' 1 , Mahsa Jahangiri-Rad ${ }^{2 *}$ (]), Hamidreza Tashauoei' ${ }^{1}$ (i) \\ 1. Department of Environmental Health Engineering, Faculty of Public Health and Biomedical Engineering, Tehran Medical Sciences, Islamic Azad \\ University, Tehran, Iran \\ 2. Water Purification Research Center, Tehran Medical Sciences, Islamic Azad University, Tehran, Iran
}

\begin{tabular}{|c|c|}
\hline $\begin{array}{l}\text { Use vour device to scan } \\
\text { and read the article online } \\
\end{array}$ & $\begin{array}{l}\text { ditation Givi M, Jahangiri-Rad M, Tashauoei HR. Assessment of Groundwater Quality in the Jajrood River Basin, Teh- } \\
\text { ran, Iran: A Coupled Physicochemical and Hydrogeochemical Study. Journal of Advances in Environmental Health Research. } \\
\text { 2021; 9(3):237-254. http://dx.doi.org/10.32598/JAEHR.9.3.1221 } \\
\text { doi) http://dx.doi.org/10.32598/JAEHR.9.3.1221 }\end{array}$ \\
\hline
\end{tabular}

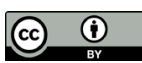

Article info:

Received: 20 Apr 2020

Accepted: 18 May 2020

Publish: 01 Jul 2021

\section{Keywords:}

Groundwater, Water quality index, Hydrogeochemistry, Subsurface soil analysis

\section{A BSTRACT}

Background: The physicochemical composition of groundwater is affected by the quantity and quality of surrounding aquifers which are in turn recharging from adjacent river waters.

Methods: In the present study, 20 surface and 16 groundwater samples were collected in pre- and post-monsoon season from the Jajrood River basin, Tehran, Iran. The samples were analyzed for 18 physicochemical water quality characteristics to assess the river and groundwater qualities. Hydrogeochemical analyses of groundwater samples were also performed to determine the Water Quality Index (WQI) for drinking and evaluate factors governing the water quality characteristic in the study area. Accordingly, the Piper diagram and Gibbs and Chadha plots were drawn to assess seasonal variations in hydrochemical facies and processes in the basin. Subsurface soil samples were also examined with respect to the structure, elemental composition, and multielemental trace analysis.

Results: Results showed the abundance of major ions in the order of $\mathrm{Ca}^{+2}>\mathrm{Na}^{+}>\mathrm{Mg}^{+2}>\mathrm{K}^{+}$for cations and $\mathrm{HCO}_{3}^{-}>\mathrm{SO}_{4}^{2-}>\mathrm{Cl}^{-}>\mathrm{NO}_{3}^{-}>\mathrm{F}^{-}$for anions. In general, all drinking groundwater samples met WHO permissible limits except for Chemical Oxygen Demand (COD) and $\mathrm{HCO}_{3}^{-}$. Moreover, the water is categorized as $\mathrm{Ca}-\mathrm{Mg}-\mathrm{HCO}_{3}$ type. Subsurface soil analyses demonstrated quartz and calcium carbonate as the main phases of soil structure, suggesting the enrichment of groundwater with temporary hardness.

Conclusion: Overall, the groundwater quality was suitable for drinking and agricultural activities.drinking and agricultural activities.

\footnotetext{
* Corresponding Author:

Mahsa Jahangiri-Rad, $P h D$.

Address: Water Purification Research Center, Tehran Medical Sciences, Islamic Azad University, Tehran, Iran.

Phone:+98 (912) 2834504

E-mail: mahsajahangiri_64@yahoo.com; m.jahangiri@iautmu.ac.ir
} 


\section{Introduction}

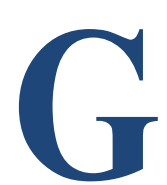

roundwater virtually accounts for half of all drinking water all over the world [1]. The quality of water through its biological content and chemical composition affects public health and agricultural production [2]. In recent decades, the quality of water bodies has decreased either by anthropogenic or natural processes, which subsequently has deteriorated its use for drinking, agriculture, and other purposes [3, 4]. The general hydrogeochemical characteristics of water regulate its use for intended purposes. Water chemistry is mainly influenced by the interaction of water with lithologic media through which it flows [5]. The chemical composition of groundwater, therefore, depends on the quality of surface water in the recharge zone and also upon various hydrogeochemical processes that happen naturally in the subsurface [6]. Seasonal variations in groundwater chemistry are also contributed to these processes. Moreover, soils have important direct and indirect impacts on water quality. A natural phenomenon, like topographic position and the mineral composition of the underlying geology, is responsible for the primary physical and geochemical conditions in groundwater that account for physical properties, such as $\mathrm{pH}$, temperature, electrical conductivity, alkalinity, and chemical concentrations of dissolved solids [7].

The chief ion profile of groundwater can provide accurate information to ascertain solute sources and delineate groundwater evolution [8, 9]. Heretofore, hydrochemical facies have been widely considered in the chemical evaluation of groundwater and surface water. In this regard, Piper [10] and Durov [11] conducted the first investigation. After that, Chadha [12] developed a simple modified Piper diagram. Many researchers have extensively used these diagrams to scrutinize the driving factors of water chemistry [13-15]. Several authors have used the Water Quality Index (WQI) to assess the water chemistry status in the river and groundwater for human consumption $[16,17]$.

WQI provides a standardized method to compare among diverse water sources based upon water quality parameters, such as common anions and cations, $\mathrm{pH}$, electrical conductivity, turbidity, fecal coliforms, etc. Based on this index, the water quality is categorized as excellent, good, medium, bad, and very bad [18]. Several studies have been conducted in Iran to understand the causes and effects of aquifers-related issues besides their current groundwater characteristics. These studies suggest that various processes influence groundwater chemistry and its impact on different beneficial uses (drinking, industrial, agricultural) [19-21]. A large and growing body of literature has investigated the surface water and groundwater interaction using hydrogeology and hydrochemical assessment. Piper, Gibbs, and Chadha diagrams have been reported as robust tools for the hydrogeochemical evaluation since the former can classify the groundwater and the latter estimates the origin of ions [22-24].

In many rural areas of Iran, wells provide a reliable and ample water supply for domestic uses. Several dug wells have recently been excavated in Pardis City, along the Jajrood river, to provide water for drinking and irrigation purposes. Drilling other wells in this area entails checking the water quality of present dug wells that are supplied by the river. The present study investigated the quality of the Jajrood river water and its effect on the physical and chemical properties of shallow wells in the study area.

An initial objective of this research was to analyze the hydrochemical characteristics of the shallow groundwater and their evolution using descriptive statistics and the Gibbs diagram. Furthermore, water types and the corresponding controlling factors of groundwater characteristics were assessed using the Piper, and Chadha approaches for the pre- and post-monsoon samples. Since the soil analyses through which the water passes provide reliable information about the chemical properties of water, we examined the structure and elements of soil samples, too. Finally, the role of subsurface soil in the chemistry of groundwater constituents was investigated.

\section{Details of the study site}

\section{Study area}

The study area is part of the Jajrood catchment in Shemiranat City based on urban political divisions (Figure 1). The geographical location of the basin is $51^{\circ} 5^{\prime}$ to $51^{\circ} 24^{\prime}$ east longitude and $35^{\circ} 46^{\prime}$ to $36^{\circ} 3^{\prime}$ north latitude. The river originates from the heights of the Alborz mountain range in the north of Tehran. The river is located $30 \mathrm{~km}$ northeast of Tehran City, the capital of Iran, with a length of $40 \mathrm{~km}$ and $710 \mathrm{~km}^{2}$ catchment area, which flows from northwest to southeast. On its way, the river supplies drinking and agricultural water for many villages, and after joining the Garmabdar branch and Ruteh river to the southeast, it enters the Latyan dam and ends at Namak (Salt) Lake. The average annual rainfall of the study area is recorded as almost $600 \mathrm{~mm}$ [25]. The decrease in temperature is observed from south to north of the research area. The average annual temperature is less than $5^{\circ} \mathrm{C}$ in the highlands and $21^{\circ} \mathrm{C}$ in the south- 
ern parts. The relative humidity of the river basin varies between $18 \%$ to $45 \%$. Pardis city, consisting of Bumhan and Jajrood districts, is in this catchment area. This city has three districts of Bumhan, Markazi, and Jajrood. The Jajrood district includes Jajrood village and Saeedabad district, where all the samples in the present study were taken.

\section{Geology and hydrology}

The general terrain of the study area consists mainly of some faults and folds to the south [25]. The soil of the area is mostly alluvial sediments and includes sand and gravel. The basin of the Jajrood river is in an area with a maximum altitude of $4000 \mathrm{~m}$ above sea level. The area can be divided into two parts in terms of elevation: 1) the mountainous area, which forms a smaller part of the Jajrood river, encompasses the continuation of the Central Alborz mountain range along with hills part of which stretches into a forest, and 2) the plain area, which is the large part of the river's catchment area. The latter is not very high, and only in some areas, little vegetation can be observed.

\section{Materials and Methods}

\section{Field survey and chemical analysis}

Twenty surface water and 16 groundwater samples were taken in pre- and post-monsoon seasons from 5 points spread over the Jajrood river basin and 4 wells recharging from the river (Figure 1). Sampling was done twice from each point, and the average results were reported. For chemical analysis, water samples were collected in polyethylene bottles. The sampling bottles were soaked with $\mathrm{HCl}$ solution then washed thoroughly twice before sampling. $\mathrm{pH}$ and Electrical Conductivity (EC) were measured in situ by portable digital meters. For cation analysis, the samples were filtered through a $0.45 \mu$ cellulose acetate membrane, and nitric acid was added to the bottles to reach a $\mathrm{pH}$ below 2 . All samples were refrigerated and analyzed within 12 hours. The sampling was followed based on standard protocols prescribed by American Public Health Association (APHA) [26]. The measurements were repeated twice, and the arithmetic average of the two measurement results was reported.

Bacteriological analysis was conducted in the same river and groundwater samples of the study area in pre- and post-monsoon seasons. The sodium thiosulfate rinsed bottles which were further sterilized in an incubator, were used for microbial sample collection. The 9-tube fermentation technique was used, and the total number of coliforms was determined as the most probable number per $100 \mathrm{~mL}(\mathrm{MPN} / 100 \mathrm{~mL})$. The analytical methods for each parameter are summarized in Table 1. Piper pilot (Piper, 1944) and Chadha's (1999) approach were applied to determine groundwater types of the study area. Gibbs (1970) diagram was used to establish the relationship of water composition and aquifer lithological characteristics [27]. All diagrams were prepared using Microsoft Excel v. 2013.

\section{Water Quality Indexes}

WQI expresses as a rating that reflects the mixed influence of various water quality parameters [28]. To calculate this index, we assigned each water quality parameter an assorted weight (wi) on a scale of 1 to 5 based on the importance of their health effects and the significance in drinking water quality (Table 2). High weight values are assigned to parameters that, if exceeded by the standard limits, have severe health effects and make the water unusable for drinking purposes [29]. The relative weight (Wi) is computed from the Equation 1 [30]:

$$
\text { (1) } W_{i}=\frac{w_{i}}{\sum^{w i}}
$$

where, Wi represents weight, wi is the weight of each parameter and shows the sum of all parameters included in the WQI calculation.

Then, a quality rating (qi) for each parameter was obtained by dividing its concentration (Ci) by its limit values ( $\mathrm{Si}$ ) given in Table 2 based on Equation 2 [17].

$$
\text { (2) } q_{i}=\frac{C_{i}}{S_{i}} \times 100
$$

To calculate WQI, the SIi value was calculated according to Equation 3:

(3) $S I_{i}=W_{i} \times q_{i}$

Ultimately, WQI was computed by Equation 4 [17].

(4) $W Q I=W Q I=\sum_{i=I}^{n} S I_{i}$

Sodium percentage $(\mathrm{Na} \%)$, sodium absorption ratio (SAR), and residual sodium carbonate (RSC) were computed to investigate the groundwater suitability of Gw2 for irrigation purposes. The concentration of groundwater samples in the following equations is presented in meq/L.

SAR was obtained from the Equation 5 [31].

(5) $S A R=\frac{N a}{\sqrt{\frac{C a+M g}{2}}}$

$\mathrm{Na} \%$ was computed using Equation 6 [32]: 
Table 1. Sampling analysis techniques

\begin{tabular}{|c|c|}
\hline Parameters & Methods \\
\hline Cations & Induced Coupled Plasma (ICP) \\
\hline Anions & Ion Chromatograph (IC) \\
\hline Alkalinity & Acid titration \\
\hline Total Hardness (TH) & EDTA titration \\
\hline Total Dissolved Solids (TDS) & $\begin{array}{l}\text { Mathematical calculations (using anions and cations concentra- } \\
\text { tion) }\end{array}$ \\
\hline $\mathrm{pH}$ & Electrometric \\
\hline Turbidity & Nephelometry \\
\hline Electrical conductivity (EC) & Conductivity meter \\
\hline $\mathrm{Zn}$ & Polarography \\
\hline Chemical Oxygen Demand (COD) & Spectrometry \\
\hline Coliforms & 9- tubes (Multi-pipe fermentation) \\
\hline
\end{tabular}

Table 2. Descriptive Statistics of parameters and the values used for Water Quality Index (WQI) calculation

\begin{tabular}{|c|c|c|c|}
\hline Parameters & WHO (2011) & Weight (wi) & Relative Weight (Wi) \\
\hline $\mathrm{pH}$ & $6.5-8.5$ & 4 & 0.085 \\
\hline EC & 1000 & 4 & 0.085 \\
\hline TDS & 500 & 5 & 0.106 \\
\hline $\mathrm{TH}$ & 200 & 2 & 0.042 \\
\hline $\mathrm{F}$ & 1.5 & 5 & 0.106 \\
\hline $\mathrm{Cl}$ & 250 & 3 & 0.063 \\
\hline $\mathrm{SO}_{4}$ & 250 & 4 & 0.085 \\
\hline $\mathrm{HCO}_{3}$ & 120 & 3 & 0.063 \\
\hline $\mathrm{NO}_{3}$ & 50 & 5 & 0.106 \\
\hline $\mathrm{Ca}$ & 300 & 2 & 0.042 \\
\hline $\mathrm{Mg}$ & 30 & 2 & 0.042 \\
\hline $\mathrm{Na}$ & 200 & 2 & 0.042 \\
\hline $\mathrm{K}$ & 12 & 1 & 0.021 \\
\hline COD & 10 & 4 & 0.085 \\
\hline $\mathrm{Zn}$ & 3 & 1 & 0.021 \\
\hline- & - & 47 & 1 \\
\hline
\end{tabular}

EC: Electrical Conductivity; TDS: Total Dissolved Solids; TH: Total Hardness; COD: Chemical Oxygen Demand. 

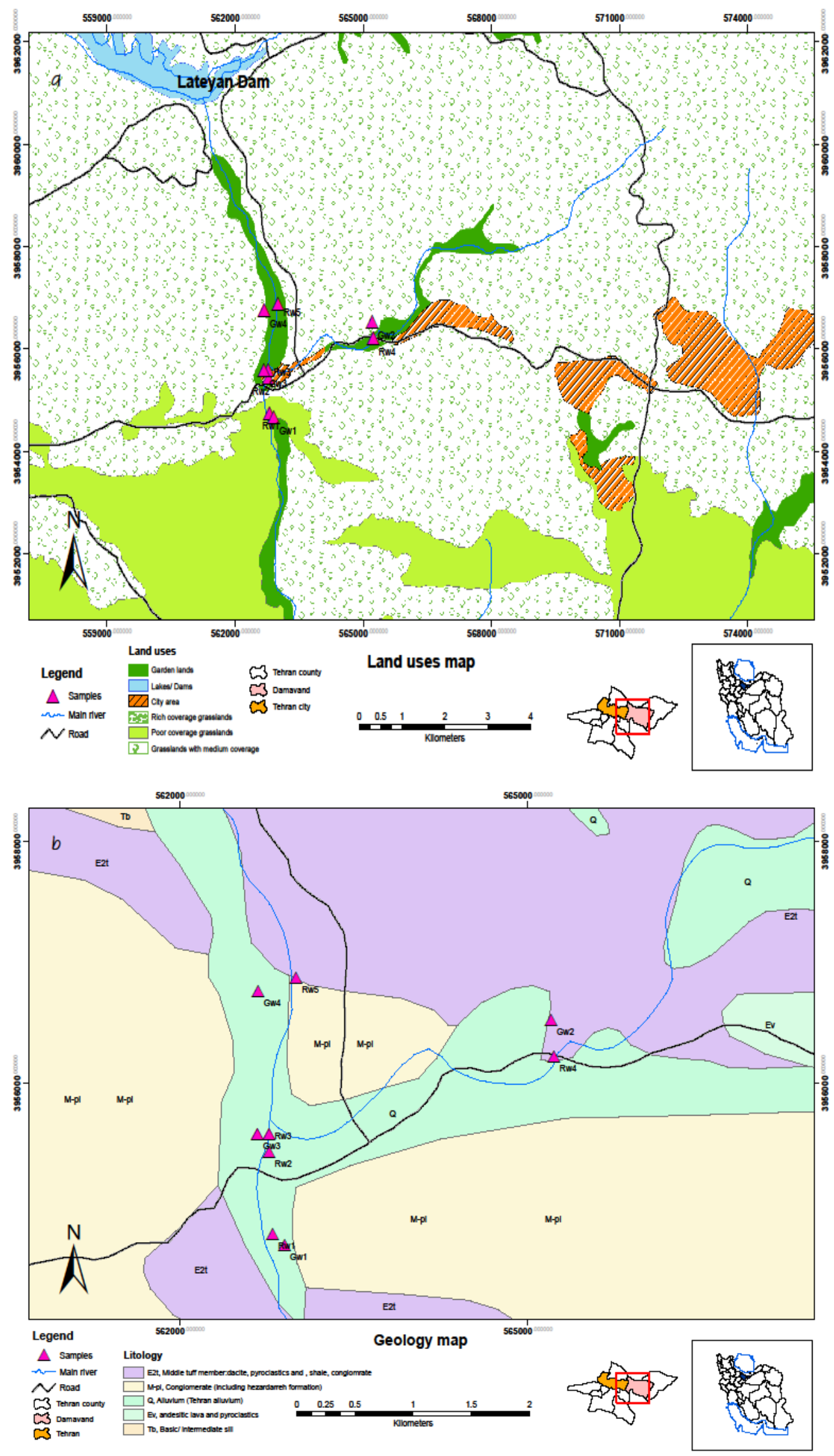

Figure 1. Sampling locations/land uses map (a) and geology map (b) of the study area 
(6) $N a \%=\frac{(N a+K) x 100}{C a+M g+N a+K}$

RSC was calculated with Equation 7 [33].

(7) $\mathrm{RSC}=\left(\mathrm{HCO}_{3}+\mathrm{CO}_{3}\right)-(\mathrm{Ca}+\mathrm{Mg})$

\section{Soil collection and analysis}

The subsurface soil sample was taken from the 12-m depth of a new excavated well in the same area. The sample was placed in a plastic bag and sent to the laboratory in the shortest time. The obtained sample was completely ground, then dried in the oven, and digested in four acids (hydrochloric acid, nitric acid, hydrofluoric acid, sulfuric acid) [34, 35]. The level of the elements is determined by the amount of dry matter in the sample. The structure and elemental compositions of the soil sample were analyzed using X-Ray Diffraction (XRD), X-Ray Fluorescence spectrometry (XRF), and inductively coupled plasma optical emission spectroscopy (ICP-OES). The ICP-OES test was performed with a German device (Arcos model). Calibration of the device was performed by providing a standard sample for the studied elements in different concentrations. The XRF test was carried out by a German Spectroscopy device (XEPOS model). The experiment was achieved in Turboquant mode using four secondary anodes, and the calculations were done based on the general standard sample. The XRD test was performed with a device from a German company (Stoe company, Stadi P model) using a copper lamp.

\section{Results and Discussion}

Groundwater quality evaluation based on the characteristics of surface water sources

The chemical composition of the groundwater and river water samples during pre- and post- monsoon seasons are summarized in Table 3 . The $\mathrm{pH}$ in river water samples ranged from 8.09 to 8.16 and 7.92 to 8.27 in preand post-monsoon seasons, respectively, indicating neutral nature and slight alkalinity of surface water, which were similar to the corresponding $\mathrm{pH}$ values of 7.33 to 7.59 and 7.2 to 7.9 observed in groundwater samples recharging from the surface points. However, the $\mathrm{pH}$ content of groundwater was slightly lower than their surface values suggesting the dissolution of $\mathrm{CO}_{2}$ in subsurface aquifers. All the groundwater samples showed the $\mathrm{pH}$ values within the permissible limit of 6.5-8.5 30.

The Electrical Conductivity (EC), which depicts the enrichment of dissolved solids in groundwater, varied from 431 to $2230 \mu \mathrm{mho} / \mathrm{cm}$ in the dry season, which was remarkably higher than their corresponding river water samples, indicating the cation-anion exchange through water-rock interaction [36]. Surprisingly, a reverse trend was noticed in the wet season as the amount of EC was lower in groundwater than the detected levels of surface water sampling points. This result may be explained by the fact that the soil is saturated during the wet season. Because of the soil reduction in ion exchange capacity, the concentration of ions decreases, which subsequently ended in a decrease in EC values.

Compared to the dry period, the EC values in both river and groundwater samples are somewhat increased in the wet season, which can be explained by a drop in the river flow resulting from the closing of the upper dam (Latyan dam) gates. Correspondingly, this effect led to a remarkable increase in the concentration of other tested parameters in all sampling points during the wet season (Table 3 ). The EC values of groundwater for drinking purposes (Gw1, Gw3, and Gw4) lowered the permissible limit of 1000 $\mathrm{mho} / \mathrm{cm}$ in both seasons. 30 High values of EC (2230 $\mathrm{mho} / \mathrm{cm}$ ) were observed in the sampling point of Gw2, which is in an agricultural land area and near a village. The enrichment of salts in Gw2 with the EC values of 2230 and $2500 \mathrm{mho} / \mathrm{cm}$ during pre- and post-monsoon seasons shows that it falls under the medium salt enrichment class (EC: $1500-3000 \mathrm{mho} / \mathrm{cm}$ ) [37].

The Total Dissolved Solids (TDS) ranged from 230.8 to $300.7 \mathrm{mg} / \mathrm{L}$ and 284.8 to $321.8 \mathrm{mg} / \mathrm{L}$ in dry and wet seasons, respectively, for the wells with drinking water purposes. These wells fall into freshwater categories (TDS $<1000 \mathrm{mg} / \mathrm{L}$ ) [30]. The measured TDS contents were 1294.9 and $1412.6 \mathrm{mg} / \mathrm{L}$ for Gw2 at each season which illustrates the brackish water category (1000$10000 \mathrm{mg} / \mathrm{L}$ ) [38]. None of the samples taken from Gw1, Gw3, and Gw4 showed TDS values exceeding the permissible limit of $500 \mathrm{mg} / \mathrm{L}$ for potable use. Accordingly, all groundwater samples in the dry season exhibited higher TDS values than those obtained from river samples. However, this phenomenon completely reversed in the post-monsoon season so that the TDS amounts in surface water exceeded groundwater levels. Gw1 in the dry season and Gw1, Gw3, and Gw4 in the wet season recorded higher Total Hardness Values (TH) beyond the permissible value of $200 \mathrm{mg} / \mathrm{L}$ as $\mathrm{CaCO} 3$ and the total alkalinity values were within the safe range of $20-200 \mathrm{mg} / \mathrm{L}$ as $\mathrm{CaCO}_{3}$ for potable water [30].

The maximum reported sample of TDS and total alkalinity were from Gw2 with irrigating purposes. $\mathrm{TH}$ values were comparatively higher than alkalinity in all water samples, illustrating non-carbonate hardness also 
Table 3. River and groundwater quality in the study area

\begin{tabular}{|c|c|c|c|c|c|c|c|c|c|c|c|c|c|c|c|c|c|c|c|}
\hline 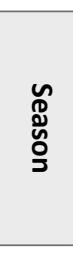 & 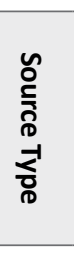 & 모 & $\frac{\pi}{\underline{Q}}$ & 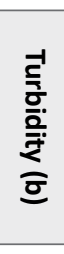 & $\frac{\vec{n}}{a}$ & $\frac{\overrightarrow{1}}{\underline{a}}$ & 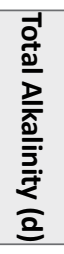 & $\frac{\pi}{a}$ & $\frac{0}{a}$ & $\frac{\tilde{0}}{a}$ & 甹 & $\frac{z}{a}$ & $\begin{array}{l}\frac{\partial}{5} \\
\frac{\sigma}{a}\end{array}$ & $\begin{array}{l}\frac{3}{o g} \\
\frac{\pi}{a}\end{array}$ & $\frac{z}{\underline{\underline{t}}}$ & $\frac{\bar{T}}{\frac{\pi}{a}}$ & $\frac{8}{a}$ & $\frac{\pi}{D}$ & $\frac{N}{a}$ \\
\hline \multirow{9}{*}{ 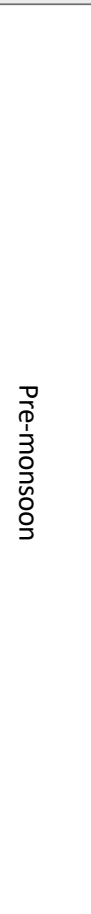 } & $\sum_{\xi}^{0}$ & $\underset{\sim}{\sim}$ & 岕 & $\stackrel{\circ}{\dot{v}}$ & 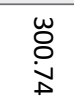 & $\widetilde{\infty}$ & : & 芯 & $\tilde{H}$ & $\underset{\omega}{\stackrel{\leftrightarrow}{\omega}}$ & 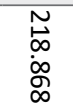 & $\ddot{\infty}$ & 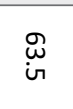 & $\underset{\infty}{\vec{⿱}}$ & $\tilde{o}$ & $\tilde{D}$ & $\checkmark$ & $\hat{\omega}$ & ¿े \\
\hline & 蛋 & $\stackrel{\substack{\infty \\
\dot{\infty}}}{ }$ & $\stackrel{\text { w }}{\text { }}$ & $\stackrel{\vec{D}}{\vec{D}}$ & 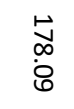 & 点 & $\vec{s}$ & $\stackrel{\circ}{\stackrel{\vec{E}}{*}}$ & $\vec{t}$ & $\tilde{o}$ & 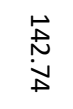 & $\underset{\sim}{\omega}$ & $\underset{i}{\overrightarrow{0}}$ & $\stackrel{\infty}{\rightarrow}$ & $\forall$ & 家 & ڤ̆ & $\tilde{\infty}$ & $\dot{\circ}$ \\
\hline & 焉 & 吕 & $\stackrel{w}{\sim}$ & $\ddot{\jmath}$ & $\begin{array}{l}\widetilde{o} \\
\mathbb{0} \\
\overrightarrow{0}\end{array}$ & 突 & $\stackrel{\vec{\sigma}}{.}$ & 总 & $\vec{v}$ & $\underset{\omega}{\omega}$ & 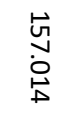 & $\underset{\dot{D}}{w}$ & 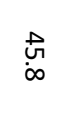 & $\stackrel{0}{\circ}$ & 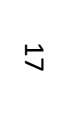 & $\vec{\bullet}$ & 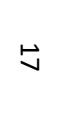 & 䓀 & $\stackrel{\circ}{\circ}$ \\
\hline & $\sum_{\omega}^{刃}$ & 嗯 & $\stackrel{\omega}{\sharp}$ & $\ddot{\vdots}$ & 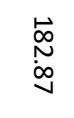 & 点 & 岕 & $\stackrel{\circ}{\stackrel{\$}{\$}}$ & 㤩 & ๘ூ & 岕 & $\omega$ & $\underset{D}{\stackrel{D}{S}}$ & $\stackrel{\infty}{\infty}$ & $\ddot{\forall}$ & $\dot{i}$ & $\stackrel{b}{ }$ & $\hat{\omega}$ & $\stackrel{\circ}{8}$ \\
\hline & 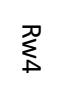 & $\stackrel{\infty}{\sim}$ & 芯 & 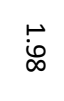 & 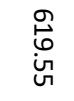 & ज্ & $\tilde{\mathcal{N}}$ & $\begin{array}{l}\stackrel{P}{\infty} \\
\stackrel{\infty}{0}\end{array}$ & $\stackrel{\vec{N}}{N}$ & $\begin{array}{l}\text { 总 } \\
\text { in }\end{array}$ & 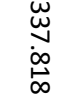 & $\underset{\infty}{\stackrel{\leftrightarrow}{\infty}}$ & ๙ૅ & 㤐 & $\stackrel{\infty}{+}$ & $\omega$ & $\tilde{N}$ & 㟧 & $\dot{8}$ \\
\hline & 亮 & w & $\tilde{\tilde{u}}$ & i & 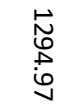 & 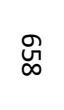 & 总 & نे & 点 & $\begin{array}{l}\text { 岁 } \\
\text { in }\end{array}$ & $\underset{\vec{D}}{\stackrel{\vec{D}}{:}}$ & $\stackrel{\omega}{\omega}^{\omega}$ & $\underset{\sim}{\stackrel{\vec{N}}{*}}$ & $\stackrel{\infty}{\infty}$ & 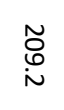 & $\stackrel{\omega}{\omega}$ & 㟔 & $\hat{\omega}$ & $\stackrel{\circ}{9}$ \\
\hline & 胥 & $\begin{array}{l}\infty \\
\stackrel{\infty}{\circ}\end{array}$ & $\stackrel{⿱ \varpi 灬}{\uplus}$ & in & $\begin{array}{l}\text { క్ } \\
\text { 心 }\end{array}$ & 点 & $\vec{v}$ & $\stackrel{\circ}{\stackrel{0}{\infty}}$ & $\underset{:}{\stackrel{D}{\circ}}$ & & 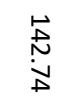 & $\omega$ & $\underset{⿱ 亠 凶}{\vec{D}}$ & $\stackrel{\infty}{\rightarrow}$ & b & 它 & 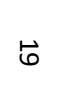 & $\widetilde{\sigma}$ & $\dot{8}$ \\
\hline & $\underset{w}{\stackrel{9}{(}}$ & ì & $\underset{બ}{\stackrel{\rightleftarrows}{~}}$ & P & 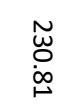 & 빙 & 岕 & 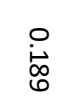 & 点 & $\underset{\sigma}{\omega}$ & 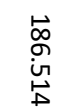 & $\stackrel{P}{\vec{D}}$ & i⿱ & $\stackrel{\vec{c}}{i}$ & $\begin{array}{l}\overrightarrow{6} \\
\infty\end{array}$ & $\tilde{\omega}$ & $\overleftrightarrow{\omega}$ & $\hat{\omega}$ & $\stackrel{\circ}{\dot{\circ}}$ \\
\hline & $\stackrel{Q}{Q}$ & $\underset{\infty}{\stackrel{w}{w}}$ & 悹 & 郘 & 芯 & ज & 岕 & 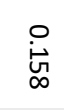 & $\widetilde{o}$ & $\underset{\omega}{\omega}$ & 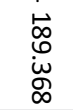 & $\sigma$ & $\underset{\tilde{\omega}}{\tilde{W}}$ & : & $\approx$ & $\underset{\infty}{+}$ & $\not$ & $\hat{\omega}$ & : \\
\hline \multirow{10}{*}{ 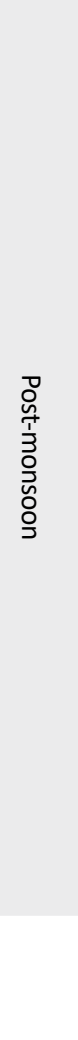 } & $\sum_{!}^{0}$ & $\ddot{\circ}$ & $\stackrel{\leftrightarrow}{\circ}$ & $\stackrel{\triangleright}{\dot{B}}$ & 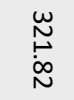 & $\widetilde{\widetilde{N}}$ & $\stackrel{\stackrel{\varpi}{\omega}}{ }$ & $\stackrel{\stackrel{\stackrel{N}{v}}{*}}{ }$ & $\stackrel{\omega}{\omega}_{i}^{\omega}$ & $\underset{\sim}{\stackrel{g}{N}}$ & 芯 & $\sigma$ & $\stackrel{g}{\sim}$ & $\underset{i \sim}{\vec{\omega}}$ & $\stackrel{\omega}{\omega}$ & $\stackrel{\vec{v}}{v}$ & $\infty$ & $\hat{\omega}$ & P \\
\hline & 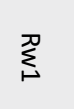 & $\stackrel{\infty}{\sim}$ & 8 & $\stackrel{\vec{v}}{v}$ & $\underset{\stackrel{w}{w}}{\underbrace{\infty}_{\infty}}$ & $\stackrel{\widetilde{\infty}}{\tilde{\infty}}$ & 鸟 & $\tilde{\tilde{o}}$ & b & $\stackrel{\sim}{\tilde{N}}$ & 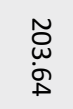 & ö & 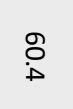 & 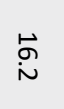 & 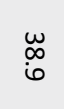 & $\tilde{N}$ & $\infty$ & $\stackrel{\mathscr{L}}{\omega}$ & $\dot{\dot{\sim}}$ \\
\hline & 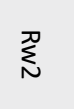 & ì & $\overrightarrow{\mathrm{s}}$ & $\underset{\sim}{\stackrel{d}{~}}$ & $\begin{array}{l}\stackrel{8}{:} \\
\text { D. }\end{array}$ & $\stackrel{w}{\sim}$ & $\tilde{o}$ & 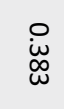 & $\stackrel{\infty}{\stackrel{\infty}{\omega}}$ & $\stackrel{\vec{v}}{\mathrm{v}}$ & 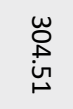 & $\stackrel{\vec{v}}{i}$ & $\vec{H}$ & $\stackrel{\omega}{\stackrel{\omega}{N}}$ & 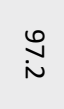 & $\stackrel{\omega}{\sigma}$ & $\tilde{\nexists}$ & $\hat{\omega}$ & $\stackrel{\triangleright}{\dot{b}}$ \\
\hline & 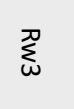 & $\stackrel{\infty}{\sim}$ & t & $\begin{array}{l}\text { Un } \\
\stackrel{\infty}{\infty}\end{array}$ & 芯 & ్ㅗ & $\stackrel{\varpi}{\uplus}$ & $\underset{\substack{\dot{0} \\
\dot{\phi}}}{ }$ & 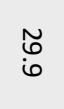 & 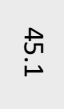 & 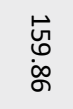 & $\stackrel{\sim}{N}$ & 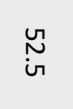 & $\stackrel{\vec{b}}{0}$ & $\stackrel{\tilde{N}}{\sim}$ & $\vec{\bullet}$ & $\hat{\theta}$ & $\tilde{\sigma}$ & $\stackrel{\text { i }}{\dot{\infty}}$ \\
\hline & $\stackrel{\prod}{\prod_{\perp}^{\prime}}$ & $\stackrel{\infty}{\stackrel{\infty}{\omega}}$ & $\overrightarrow{\mathrm{o}}$ & $\underset{్}{w}$ & $\begin{array}{l}\text { un } \\
\text { o } \\
\text { is }\end{array}$ & 岕 & $\underset{\infty}{\widetilde{\infty}}$ & 竎 & $\stackrel{M}{\rightleftarrows}$ & 䓌 & 芯 & $\overrightarrow{i n ~}_{i}$ & 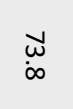 & 岕 & 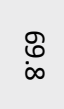 & $\underset{v}{\omega}$ & $\underset{\sim}{\sim}$ & $\hat{\omega}$ & ï \\
\hline & 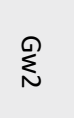 & 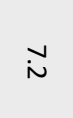 & 行 & $\tilde{\tilde{N}}$ & 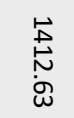 & خે & 志 & 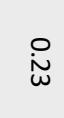 & 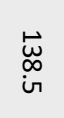 & హี & 怘 & $\underset{+}{\stackrel{\leftrightarrow}{+}}$ & 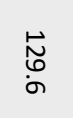 & 莕 & 岕 & $\sim$ & $\hat{\imath}$ & $\hat{\omega}$ & $\stackrel{i}{i}$ \\
\hline & 蛋 & $\stackrel{\infty}{\mathcal{N}}$ & 点 & $\begin{array}{l}\vec{D} \\
\text { D. }\end{array}$ & $\underset{\substack{\tilde{i} \\
\text { N }}}{\tilde{N}}$ & $\stackrel{\vec{\omega}}{\vec{\omega}}$ & $\stackrel{\breve{w}}{.}$ & $\stackrel{\text { 㐫 }}{\sim}$ & $\tilde{o}$ & ज & $\stackrel{\stackrel{\leftrightarrow}{g}}{\dot{y}}$ & $\stackrel{\sim}{\omega}$ & $\stackrel{\sim}{5}$ & $\stackrel{5}{0}$ & $\underset{\tilde{\sigma}}{\tilde{\sigma}}$ & $\vec{\bullet}$ & 台 & f & $\stackrel{\circ}{\circ}$ \\
\hline & 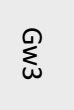 & $\ddot{g}$ & 怘 & i & 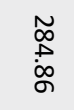 & 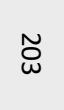 & 它 & $\begin{array}{l}\stackrel{\mathscr{N}}{\tilde{\sigma}}\end{array}$ & $\underset{i}{N}$ & $\stackrel{N}{N}$ & $\underset{\infty}{\tilde{\infty}}$ & $\stackrel{\sim}{v}$ & $\underset{\substack{\sim \\
\dot{\infty}}}{0}$ & $\underset{\oplus}{\stackrel{\leftrightarrow}{\sim}}$ & $\widetilde{\tilde{\omega}}$ & $\tilde{H}$ & $\tilde{A}$ & $\hat{\omega}$ & $\stackrel{\circ}{\circ}$ \\
\hline & $\sum_{+}^{Q}$ & is & 哭 & $\stackrel{\overrightarrow{\dot{b}}}{\vec{b}}$ & 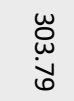 & $\tilde{\Delta}$ & 它 & 递 & $\stackrel{\infty}{\sim}_{\sim}^{w}$ & 岕 & 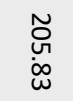 & ö & $\underset{D}{M}$ & 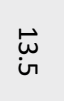 & 䓵 & 吕 & $\tilde{\sigma}$ & $\hat{\omega}$ & $\stackrel{\circ}{i}$ \\
\hline & 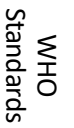 & $\begin{array}{l}\dot{0} \\
\dot{i n} \\
\dot{\phi} \\
i n\end{array}$ & 客 & & 岁 & 능 & $\begin{array}{l}\tilde{0} \\
\stackrel{\tilde{N}}{8}\end{array}$ & $\dot{\mathrm{i}}$ & ৪े & 호 & \& & II & $\tilde{8}$ & 岁 & $\tilde{8}$ & $\tilde{8}$ & ๖ & $\hat{\bullet}$ & u \\
\hline
\end{tabular}

EC: Electrical Conductivity; TDS: Total Dissolved Solids; TH: Total Hardness; COD: Chemical Oxygen Demand; FC: Fecal Coliform. 


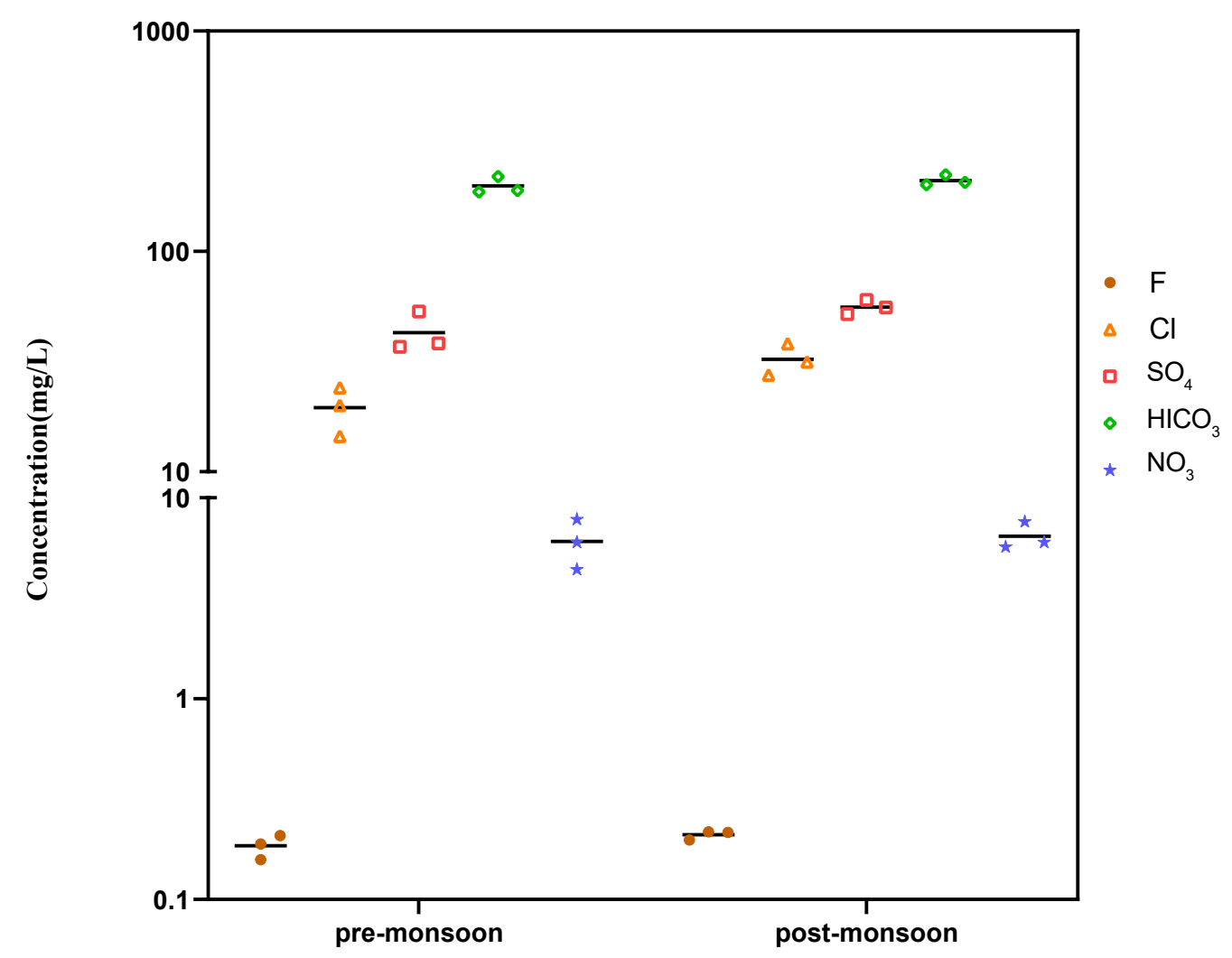

Figure 2. Detected anions concentration in groundwater during pre- and post-monsoon

exists. Overall, we found that the TH and TDS concentrations shifted post-monsoon season in surface or groundwater samples. This result might be due to the leaching of the dissolved materials with monsoon water from the unsaturated zone to the phreatic zone (saturated zone) or activation of dissociation and dissolution process that ultimately enrich waters in ionic contents. Moreover, the influence of lower flow rate during wet seasons on probable higher ions concentrations should also be considered. Ouyang et al. stated that an important parameter contributing to river water quality variation for one season might be insignificant for another season
[30]. Bicarbonate was dominant among all the anions, and its concentration slightly rose in the post-monsoon season (Figure 2).

The same trend was observed for other tested anions, as higher amounts were noticed in the wet season. Concentrations of $\mathrm{F}^{-}, \mathrm{Cl}^{-}$, and $\mathrm{NO}_{3}{ }^{-}$in groundwater samples were lower than the permissible WHO recommended values for drinking water [30], which indicates the anthropogenic sources are not highly affected the water bodies. However, some authors speculated that nitrate concentration $>5 \mathrm{mg} / \mathrm{L}$, as observed in our research, can indi-

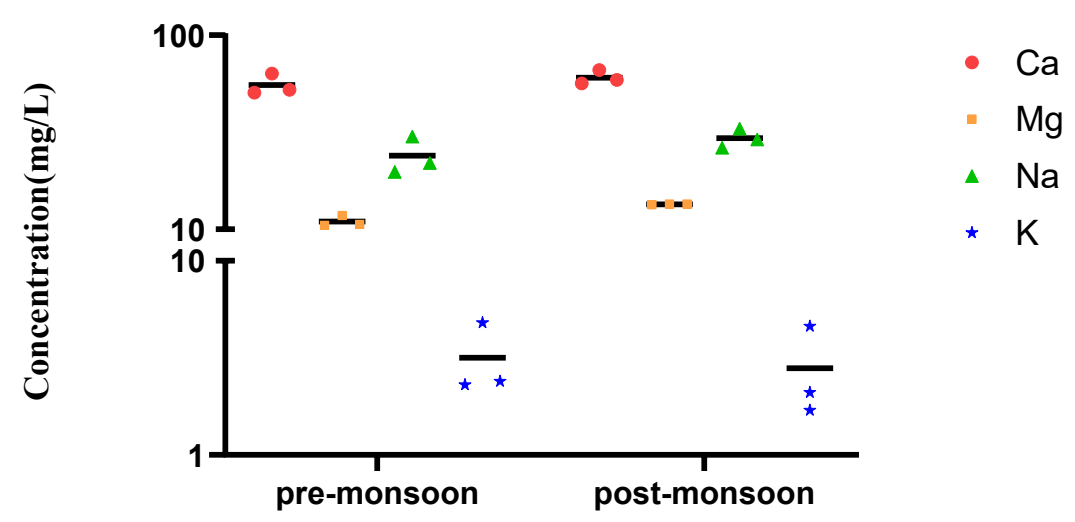

Figure 3. Detected cations concentration in groundwater samples during pre- and post-monsoon 


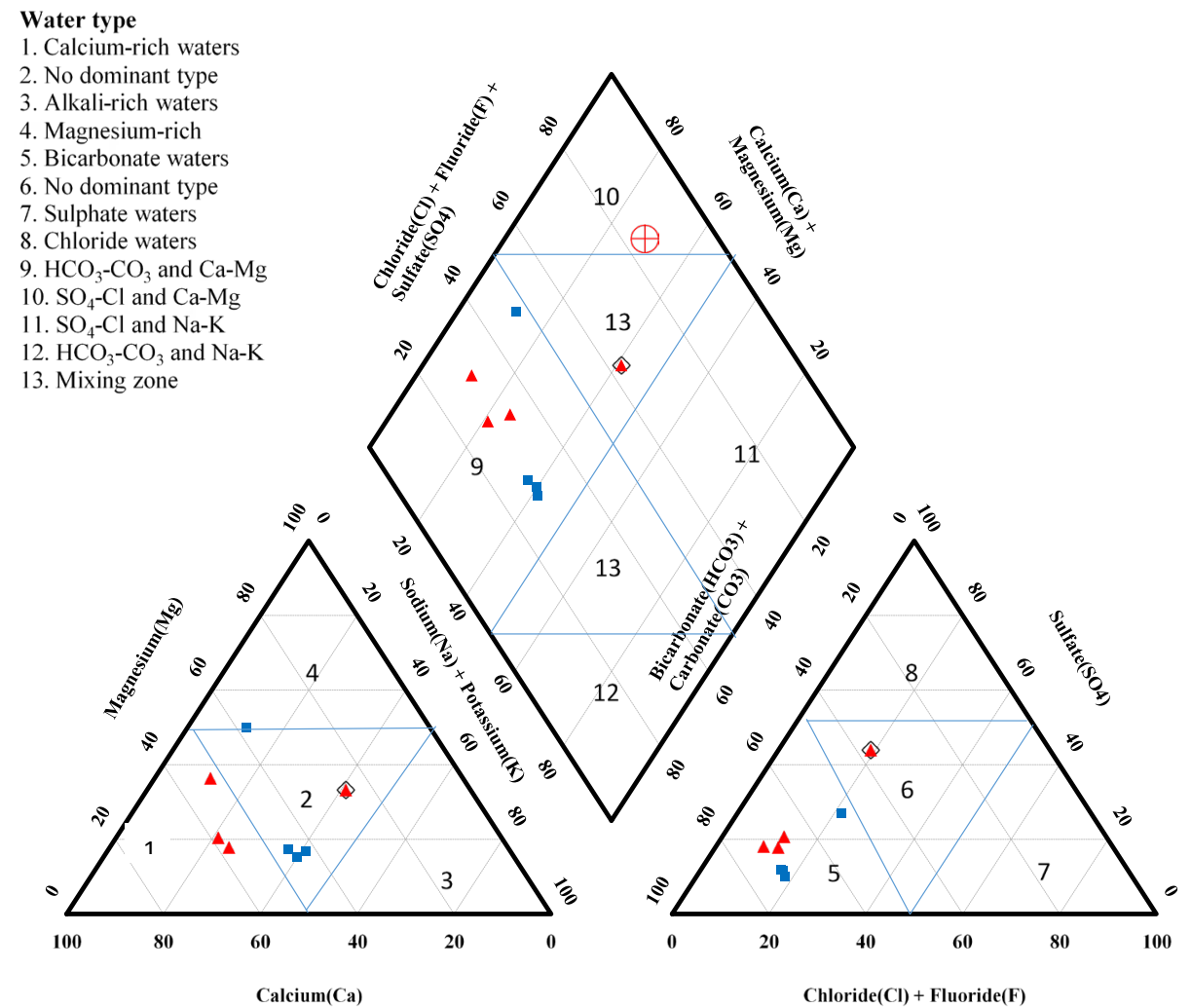

Figure 4. Piper diagrams for the chemical composition of groundwater samples

\begin{tabular}{|ll|}
\hline Water type \\
1. & $\mathrm{Ca}+\mathrm{Mg}>\mathrm{Na}+\mathrm{K}$ \\
2. & $\mathrm{Na}+\mathrm{K}>\mathrm{Ca}+\mathrm{Mg}$ \\
3. & $\mathrm{CO}_{3}+\mathrm{HCO}>\mathrm{SO}_{4}+\mathrm{Cl}$ \\
4. & $\mathrm{SO}_{4}+\mathrm{Cl}>\mathrm{CO}_{3}+\mathrm{HCO}_{3}$ \\
& \\
\hline
\end{tabular}

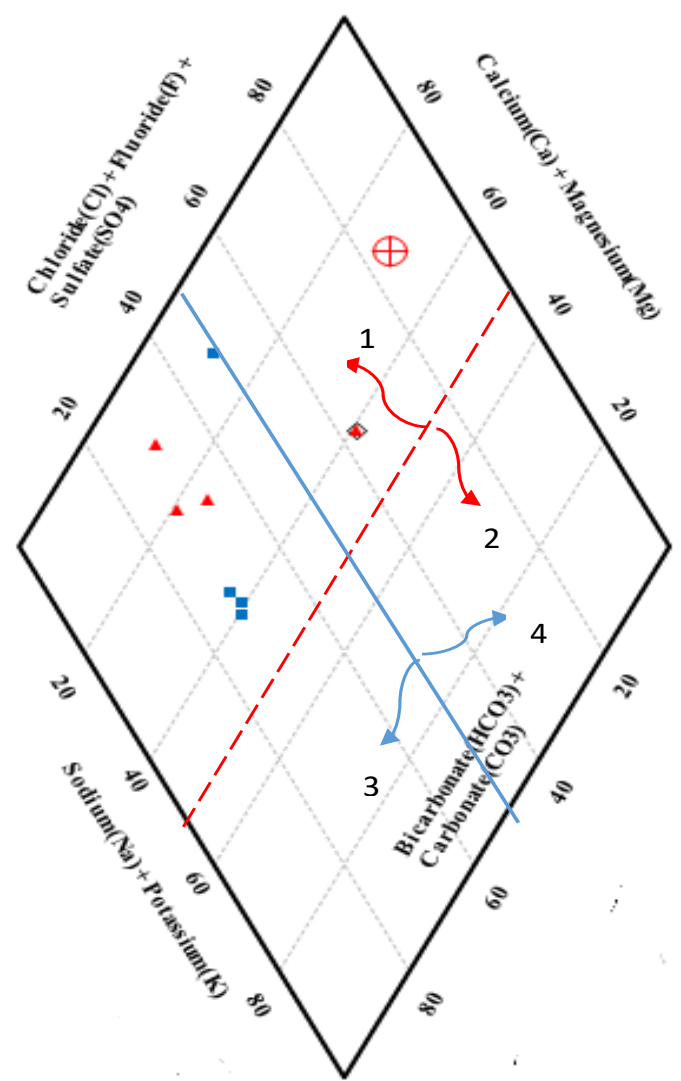

Figure 5. Hydrochemical facies plot illustrating the principal geochemical process in the study area 
(a)

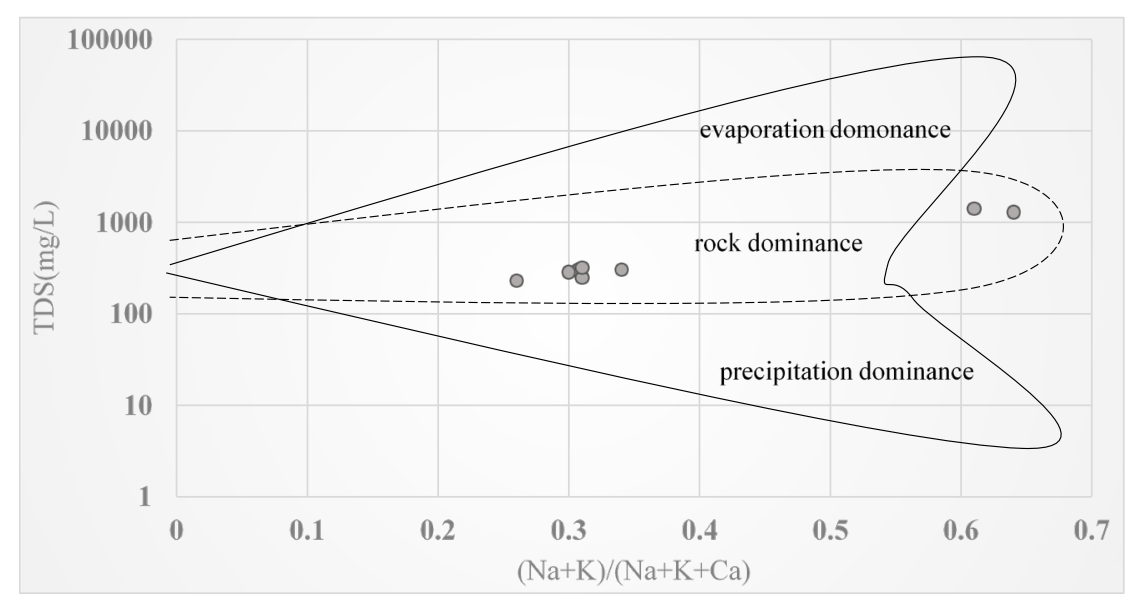

(b)

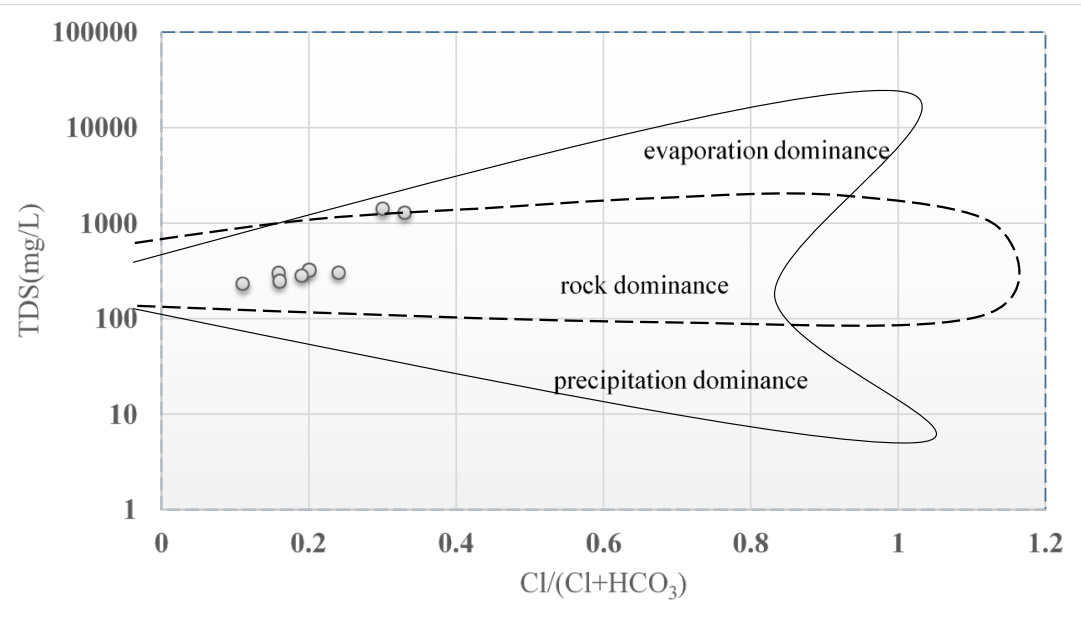

Figure 6. The ratio of cations $[(\mathrm{Na}+\mathrm{K}) /(\mathrm{Na}+\mathrm{K}+\mathrm{Ca})]$ and anions $\left[\mathrm{Cl} /\left(\mathrm{Cl}+\mathrm{HCO}_{3}\right)\right]$ against TDS

A: Gibbs plots representing the TDS versus $\mathrm{Na} /(\mathrm{Na}+\mathrm{Ca})$; and $\mathrm{B}$ : TDS versus $\mathrm{Cl} /\left(\mathrm{Cl}+\mathrm{HCO}_{3}\right)$ in both seasons.

cate water contamination by sewage, animal waste, or fertilizers $[39,40]$. The maximum concentration of $\mathrm{NO}_{3}^{-}$ in the study region was found at Gw2 (33.3 and 49.4 $\mathrm{mg} / \mathrm{L}$, in dry and wet seasons, respectively) in an agricultural area. These results might be due to the entrance of exogenous contaminants from irrigation water and or fertilizers application over the area. The application of $\mathrm{N}$-fertilizers by farmers in their fields resulted in the leaching of $\mathrm{NO}_{3}^{-}$through the soil profile and enriched local aquifers with this ion [41]. Also, $\mathrm{SO}_{4}^{2-}$ content varied from 36.9 to $53.3 \mathrm{mg} / \mathrm{L}$ and 52 to $60.2 \mathrm{mg} / \mathrm{L}$ in groundwater supplying drinking water, and all were within the permissible range as per by WHO (400 mg/L) [30].

As shown in Table 3, the sulfate concentration in the suppliers' river water remained relatively stable in various sampling points. Only one sample (Gw2) demonstrated a high concentration of $\mathrm{SO}_{4}^{2-}$ which serves for agricultural irrigation. Higher levels of $\mathrm{SO}_{4}{ }^{2-}$ and $\mathrm{Cl}^{-}$in Gw2 might be due to the interaction with a saline layer or leakage of irrigation return water [42]; also, its contamination might be related to the proximity to rural areas and contamination with domestic sewage. Sulfate mineral dissolution and sulfide mineral oxidation have been known as the natural sources causing excess $\mathrm{SO}_{4}^{2-}$ in groundwater [43]. Some authors have speculated anthropogenic sources of $\mathrm{SO}_{4}^{2-}$ rather than geogenic origin based on low anhydrite saturation index [44]. In this regard, atmospheric deposition, sulfate-containing fertilizers, bacterial oxidation of sulfur compounds, and other human impacts may result in $\mathrm{SO}_{4}^{2-}$ high values in groundwater [45]. Importantly, all groundwater samples are deficient in minimum fluoride content as recommended by WHO of $0.7 \mathrm{mg} / \mathrm{L}$ to prevent dental caries [18]. Therefore, people who use these groundwater sources may be 
at risk of dental caries. The concentration of $\mathrm{HCO}_{3}^{-}$was high in the study area, and all groundwater samples exceeded the WHO standard limits of $120 \mathrm{mg} / \mathrm{L}$ for bicarbonate. Appello and Postma speculated that chemical reaction between groundwater and silicate minerals is the main cause of $\mathrm{HCO}_{3}^{-}$formation in groundwater. Carbonate-rich rocks, such as crystalline limestone, dolomitic limestone, and calc-granulite, are demonstrated as the main sources for carbonate weathering [46]. Aquifers enrich with high bicarbonate levels during irrigation, rainfall infiltration, and groundwater move through these rocks. The abundance of major anions in groundwater samples was in the order of $\mathrm{HCO}_{3}^{-}>\mathrm{SO}_{4}{ }^{2-}>\mathrm{Cl}^{-}>\mathrm{NO}_{3}$ $>\mathrm{F}^{-}$. Calcium was found as the dominant cation, among others, indicating samples are calcium-rich (Figure 3). Except for Gw2, all groundwater samples fall below the permissible limit as per WHO during both seasons [30]. As shown in Figure 3, the groundwater concentrations of $\mathrm{Ca}^{2+}, \mathrm{Mg}^{2+}$, and $\mathrm{Na}^{+}$increased in the post-monsoon season though $\mathrm{K}^{+}$levels decreased moderately at the same season compared with the pre-monsoon season. The order of major cations abundance was $\mathrm{Ca}^{2+}>\mathrm{Na}^{+}>\mathrm{Mg}^{2}+$ $>\mathrm{K}^{+}$. A rapid increase in the major ion concentration was found in groundwater during the pre-monsoon season. Overall, $\mathrm{Na}+$ concentration was higher than $\mathrm{K}^{+}$values and almost exceeded $\mathrm{Mg}^{2+}$ concentration in the study area. A possible explanation for these results may be the cation exchange process during which $\mathrm{Na}^{+}$replaces $\mathrm{Mg}^{2+}$ and $\mathrm{K}^{+}$as the water penetrates through the soil [47].

Few studies have specifically examined the relation between $\mathrm{Ca}^{2+}$ and $\mathrm{Mg}^{2+}$ and stated that the calcium content is 3 to 4 fold higher than magnesium in natural waters [48]. In the current study, $\mathrm{Ca}^{2+}$ to $\mathrm{Mg}^{2+}$ ratio was greater than 3 in most of the examined ground and surface water samples, indicating that they are not polluted. However, this ratio was low for Rw4 that recharges well (Gw2) in both seasons, indicating that the discharges from the local households contaminate this point. Regarding sodium and potassium values, Rw4 and Gw2 were registered with high concentrations. Such elevated levels of these elements do not occur on arable lands [49], which suggests that water composition in these locations may be controlled by wastewater flowing from local households [50].

Calcium and bicarbonate ions were remarkably higher in concentration and the chief principle of elevated levels of EC in the study area. COD values of the groundwater samples ranged from 7 to 16 and $<1$ to $32 \mathrm{mg} / \mathrm{L}$ in pre- and post-monsoon seasons, respectively. Except for Gw1, other groundwater sampling points have registered COD values above the WHO provisional drinking water limit of $10 \mathrm{mg} / \mathrm{L}$ in both seasons. It suggests the possible entry of natural organic matter into surface waters that subsequently penetrate aquifers. Also, due to inorganic chemicals in water, a COD test can indicate the amount of oxygen needed to oxidize them [51]. River water demonstrated a relatively higher level of COD as compared with groundwater samples. According to Amneera et al. the elevated COD concentration illustrates polluted water [45]. COD values less than $20 \mathrm{mg} / \mathrm{L}$ exhibits unpolluted waters. The higher amounts of COD and Biochemical Oxygen Demand (BOD) were identified in the Jajrood river samples during various seasons in 2009 $[52,53]$. The authors concluded that excessive organic contaminants with different sources such as domestic, agricultural, industrial, and naturally influenced water oxygen demand. Based on field observation, excessive COD values (21 and $32 \mathrm{mg} / \mathrm{L}$ ) in Rw4 during both seasons are related to fertilizers and pesticide application over agricultural lands besides local sewage discharge.

All recorded zinc $(\mathrm{Zn})$ values in water samples were far below the limit set by international guidelines. 30 The $\mathrm{Zn}$ values were higher for surface water than their amounts in groundwater samples revealing river water pollution with zinc. The bacteriological analysis (fecal coliform) showed that the groundwater samples were not contaminated (MPN/100 mL <3). Fecal Coliform (FC) values were generally lower than river water samples demonstrating the bacteria screened out by the soil pores and rocks 51 or the bactericidal role of chlorine injected into drinking water wells [54]. Generally, the values obtained in our study are almost similar to those reported by Razmkhah et al. [48], who also emphasized that the morphology of soil, overland runoff, and fertilizers are the main sources of chemical water constituents of the Jajrood river.

\section{Groundwater quality assessment}

The water quality index has been applied to assess the quality of any water body. To calculate WQI values at each groundwater sampling point, the weight values were assigned for each water quality parameter according to their relative importance in the overall quality of water for drinking purposes. The relative weight of each parameter in the WQI calculation and their corresponding standard levels are shown in Table 2. The parameters of TDS, $\mathrm{F}^{-}$, and $\mathrm{NO}_{3}^{-}$were assigned the weight of 5; $\mathrm{pH}, \mathrm{EC}, \mathrm{SO}_{4}^{2-}$, and $\mathrm{COD}$ were assigned 4 , taking into account their health significance. $\mathrm{K}^{+}$and $\mathrm{Zn}^{+2}$ were assigned the minimum relative weight due to their least importance in water quality and health-related impacts. The computed WQI of groundwater samples for drinking 


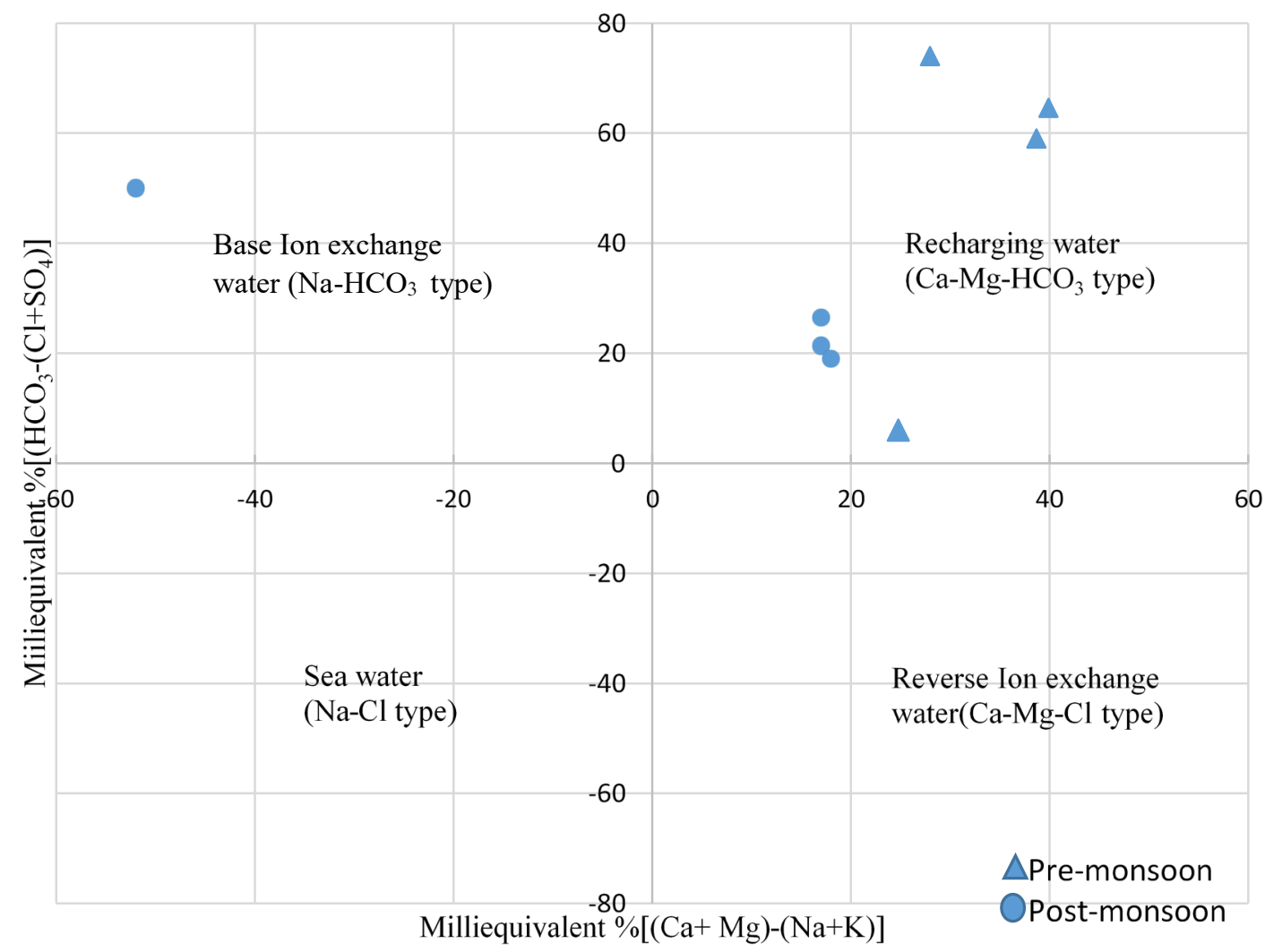

Figure 7. Chadha's plot to reveal the driving geochemical process in the study area

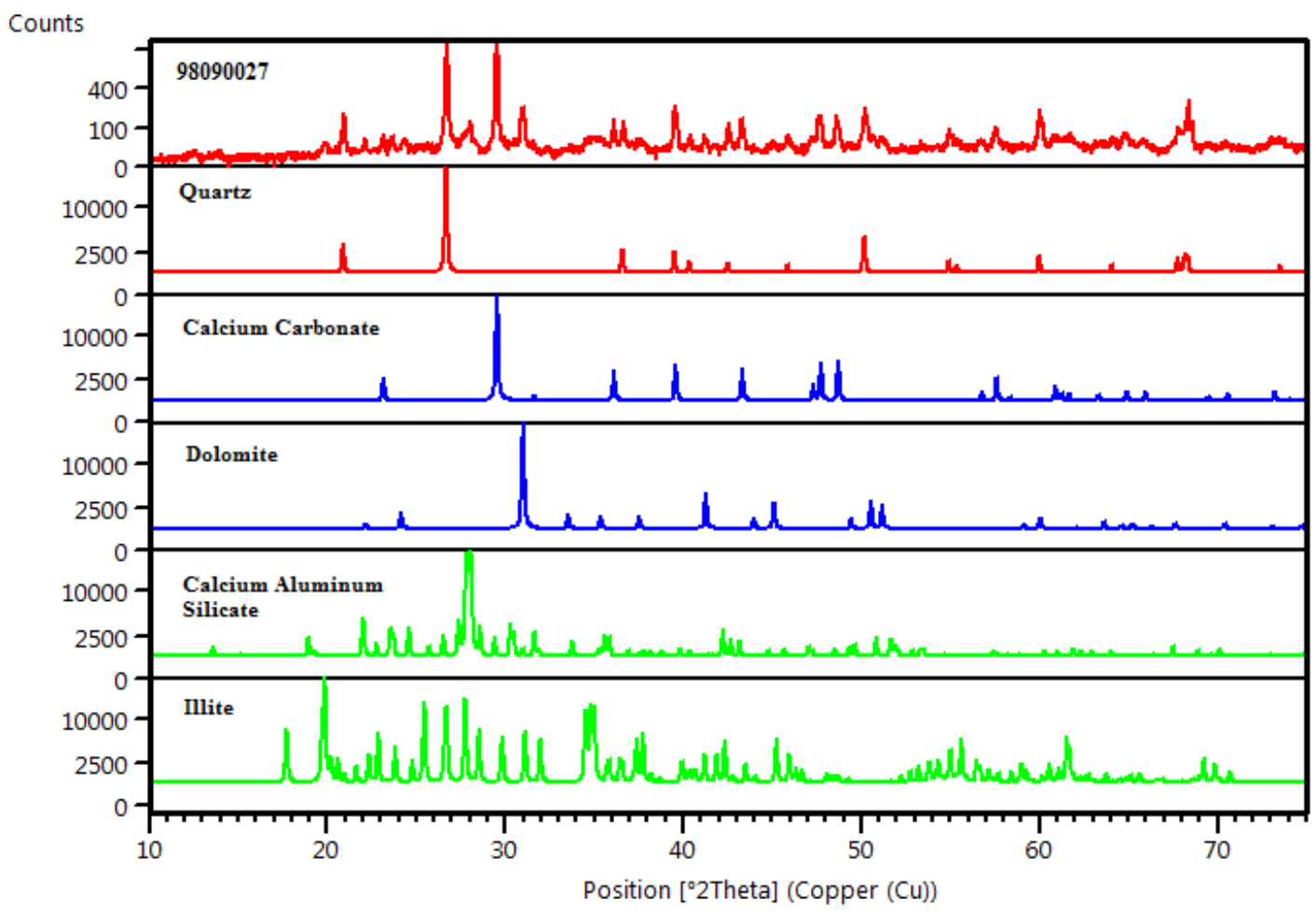

Figure 8. XRD pattern of the soil sample collected from $12 \mathrm{~m}$ depth of study area 
Table 4. Classification of groundwater based on calculated Water Quality Index (WQI)

\begin{tabular}{ccc}
\hline & \multicolumn{2}{c}{ WQI (Water Type) } \\
\cline { 2 - 3 } Sample No. & Pre-monsoon & Post-monsoon \\
\hline Gw1 & 49.36 (Excellent water) & 52.07 (Good water) \\
Gw3 & 47.00 (Excellent water) & 60.95 (Good water) \\
Gw4 & 49.32 (Excellent water) & 64.69 (Good water) \\
\hline
\end{tabular}

use ranged from 47 to 49.36 and 52.07 to 64.69 in the preand post-monsoon seasons, respectively (Table 4). Higher WQI values were observed in post-monsoon seasons as the water quality declined from "excellent" in the premonsoon to "good" in the post-monsoon season. Elevated values of WQI in the wet period were related to COD, $\mathrm{HCO}_{3}^{-}$, TDS, and $\mathrm{TH}$, which all depicted higher Standard Index (SI) values. The amount and composition of dissolved elements in water are of great importance for agricultural use.

Excessive concentrations of dissolved ions in farming water change soil configuration, permeability, and aeration, consequently hardening plant development $[55,56]$. The parameters such as sodium percentage $(\mathrm{Na} \%)$, sodium adsorption ratio (SAR), and residual sodium carbonate (RSC) are essential to determine the suitability of groundwater for agricultural use $[57,58]$. Since one of the wells (Gw2) supplies water for agricultural irrigation, and the mentioned indicators were employed to check its water suitability. RSC is used to evaluate the harmful effects of carbonate and bicarbonates on water quality for agricultural purposes [33]. Based on the calculated RSC values (-5.13 and $1.92 \mathrm{meq} / \mathrm{L})$, the well water falls into "safe" and "marginally suitable" categories in pre- and post-monsoon seasons, respectively. The negative values of RSC implied the lower amounts of $\mathrm{CO}^{2-}$ and $\mathrm{HCO}_{3}^{-}$than $\mathrm{Ca}^{2+}$ and $\mathrm{Mg}^{2+}$ levels in the groundwater. This fact may explain the absence of residual carbonate to react with $\mathrm{Na}^{+}$to worsen soil quality due to alkali hazards [56, 59]. Considering sodium, the SAR index has been applied for evaluating the suitability of irrigation water [58].
The calculated $\mathrm{Na} \%$ ( $41 \%$ and $40 \%$ ) shows that the well water was in "permissible" and "good" conditions for the same periods, respectively. The well water exhibited safe quality based on computed SAR (3.54 and 3.56) in both seasons. Overall, the estimated indexes suggest that the groundwater quality (Gw2) in the study area is suitable for agricultural purposes.

\section{Hydrogeochemical facies (piper)}

The Piper diagram was used to investigate the hydrogeochemical facies of groundwater in the Jajrood river basin. As shown in Figure 4, the water of 3 wells (Gw1, Gw3, and Gw4) fall in the zone of calcium-rich and no dominant cation type in pre- and post-monsoon seasons, respectively. Moreover, analysis of the piper diagram revealed that bicarbonate is the dominant ion and the major water type of the study area is the $\mathrm{Ca}^{2+} / \mathrm{Mg}^{2+}-\mathrm{HCO}_{3}$ (Figures 4 and 5). This finding suggests the presence of temporary hardness in the groundwater of the Jajrood basin. Figure 5 explains the superiority of alkaline earth exceeds alkali $\left(\mathrm{Ca}^{2+}+\mathrm{Mg}^{2}+>\mathrm{Na}^{+}+\mathrm{K}^{+}\right)$and weak acidic anions dominant over strong acidic anions $\left(\mathrm{HCO}_{3}{ }^{-}>\mathrm{Cl}^{-}\right.$ $\left.+\mathrm{SO}_{4}^{2-}\right)$. It is also shown in Figure 4 that all groundwater samples except Gw2 in the pre-monsoon period are in zone 9, suggesting temporary hardness is controlling the water quality. Gw2, which is in zone 9, belongs to the mixing zone, where water type can be identified as neither anion nor cation dominant. None of the samples fall under zone 10, indicating non-carbonate hardness belonging to $\mathrm{Ca}, \mathrm{Mg}^{-}, \mathrm{SO}_{4}^{2-}, \mathrm{Cl}$ does not exist. Furthermore, no samples fell under zone 11 and 12, suggesting that halite dissolution (saline) or alkali carbonate enrichment were not affecting water type [57]. According to Mechal

Table 5. Inductively Coupled Plasma (ICP) results of elements content in the investigated soil

\begin{tabular}{cccccccccccc}
\hline Type of Elements & $\mathbf{N a}$ & $\mathbf{M g}$ & $\mathbf{A l}$ & $\mathbf{S i}$ & $\mathbf{P}$ & $\mathbf{S}$ & $\mathbf{C l}$ & $\mathbf{K}$ & $\mathbf{C a}$ & $\mathbf{H g}$ \\
\hline$\%$ & 0.60 & 1.25 & 4.00 & 17.50 & 0.07 & 0.01 & 0.01 & 1.40 & 10.58 & $<0.01$ \\
\hline Type of Elements & $\mathrm{Fe}$ & $\mathrm{Co}$ & $\mathrm{Ni}$ & $\mathrm{Cu}$ & $\mathrm{Zn}$ & $\mathrm{As}$ & $\mathrm{Se}$ & $\mathrm{Cr}$ & $\mathrm{Mn}$ & $\mathrm{Cd}$ \\
\hline & 2.00 & $<0.01$ & $<0.01$ & $<0.01$ & 0.01 & $<0.01$ & $<0.01$ & 0.01 & 0.10 & $<0.01$ \\
\hline
\end{tabular}


Table 6. X-ray Fluorescence Spectrometry (XRF) analysis of the collected soil samples

\begin{tabular}{cccccccccccc}
\hline Type of Elements & $\mathbf{B a}$ & $\mathbf{C a}$ & $\mathbf{C o}$ & $\mathbf{C r}$ & $\mathbf{C u}$ & $\mathrm{Fe}$ & $\mathbf{K}$ & $\mathbf{M g}$ & $\mathbf{M n}$ & $\mathbf{N i}$ \\
\hline $\begin{array}{c}\text { Concentration } \\
(\mathrm{mg} / \mathrm{kg})\end{array}$ & 260 & 102100 & 7 & 20 & 18 & 15200 & 19000 & 11200 & 885 & 20 \\
$\begin{array}{c}\text { Type of Elements } \\
\begin{array}{c}\text { Concentration } \\
(\mathrm{mg} / \mathrm{kg})\end{array}\end{array}$ & $\mathbf{P b}$ & $\mathbf{P}$ & $\mathrm{Se}$ & $\mathrm{Zn}$ & $\mathbf{A g}$ & $\mathbf{A s}$ & $\mathbf{B i}$ & $\mathbf{C d}$ & $\mathbf{G a}$ & $\mathbf{H g}$ \\
\hline
\end{tabular}

et al. as the $\mathrm{CO}_{2}$ hydrolyzed soil silicate, $\mathrm{HCO}_{3}^{-}$releases in the groundwater. In this research, the dominance of $\mathrm{Ca}^{2+}-\mathrm{HCO}_{3}^{-}$water type illustrates the possible hydrolysis of silicate minerals by $\mathrm{CO}_{2}$ [56]. Katz et al. also stated that the $\mathrm{Ca}^{2+} / \mathrm{Mg}^{2+}$ ratio greater than 2 indicates that the hydrolysis of silicate minerals is the chief reason for high $\mathrm{Ca}^{2+}$ and $\mathrm{Mg}^{2+}$ amounts in the groundwater [57]. According to Table 1, all samples have $\mathrm{Ca}^{2+} / \mathrm{Mg}^{2+}$ ratios greater than 2 revealing the hydrolysis of silicate minerals could lead to $\mathrm{Ca}^{2+} / \mathrm{Mg}^{2+}$ release into groundwater.

The Mechanism controlling groundwater chemistry (chadha and gibbs)

Gibbs proposed the geochemistry of groundwater with respect to atmospheric precipitation, rock dissociation, and evaporation. However, Li et al. reported the downside of the Gibbs diagram. They argued that the impacts of human activities on groundwater chemistry have been ignored [59].

This diagram represents the ratio of cations $[(\mathrm{Na}+\mathrm{K}) /$ $(\mathrm{Na}+\mathrm{K}+\mathrm{Ca})]$ and anions $\left[\mathrm{Cl} /\left(\mathrm{Cl}+\mathrm{HCO}_{3}\right)\right]$ against TDS. Figure 6 specifies that most of the water samples during both periods belonged to rock dominance, with the TDS values below $1000 \mathrm{mg} / \mathrm{L}$, affecting groundwater chemical constitution. No samples were classified as precipitation or evaporation dominant, indicating the limited role of atmosphere and the dissolution of evaporates like halite $(\mathrm{NaCl})$, gypsum $\left(\mathrm{CaSO}_{4} 2 \mathrm{H}_{2} \mathrm{O}\right)$, and mirabilite $\left(\mathrm{Na}_{2} \mathrm{SO}_{4}\right)$ in the input of solutes $[60,61]$. The post-monsoon season did not alter the hydrochemical facies in the study area. Since high TDS content was accompanied by a notable increase in $\mathrm{HCO}_{3}$ concentration, the role of evaporation is negligible. The geology and environment of water affect the type and amounts of salts in water bodies [62]. Chadha proposed a hydrochemical diagram reflecting various hydrochemical processes involved in water quality [12]. The differences between alkaline earth $\left(\mathrm{Ca}^{2+}+\mathrm{Mg}^{2+}\right)$ and alkaline metals $\left(\mathrm{Na}^{+}+\mathrm{K}^{+}\right)$in meq/L for each sampling point were plotted against the difference between weak acidic anions $\left(\mathrm{HCO}_{3}{ }^{-}+\mathrm{CO}_{3}{ }^{2-}\right)$ and strong acidic anions $\left(\mathrm{Cl}^{-}\right.$ $+\mathrm{SO}_{4}^{2-}$. Then, the result is depicted in Figure 7. Chadha suggested four processes controlling the quality of water: base ion exchange water, reverse ion exchange water, recharging water, and seawater types [12]. The position of data points in Figure 7 demonstrates that recharging water was the driving process of the chemical composition of water in both seasons. Recharging waters are shaped when water penetrates the soil and dissolves carbonate and calcium in the subsurface layers. However, based ion exchange was the dominant process for one sampling point (Gw2) in the wet period, which represents the base ion exchange reaction path of groundwater. In this case, $\mathrm{Ca}^{2+}-$ $\mathrm{HCO}_{3}{ }^{-}$type water mixes with $\mathrm{Na}^{+}-\mathrm{Cl}^{-}$water type, which finally produced $\mathrm{Na}^{+}-\mathrm{HCO}_{3}^{-}$type. The high values of $\mathrm{Na}^{+}$ and $\mathrm{Cl}^{-}$for site $\mathrm{Gw} 2$ in post-monsoon (Table 1) also confirm this phenomenon. The high values of chloride ions in the water represent the influence of agrochemicals and domestic wastewaters [62]. Given that the study area is in agricultural communities, applying organic and inorganic fertilizers might pollute the groundwater by this ion.

\section{Quality in relation to subsurface soil structure and composition}

Natural mineral weathering dissolution can be the main reason for high-level EC related to dissolved solids in groundwater bodies. In this regard, some subsurface soil samples were analyzed using XRD, XRF, and ICP for the presence of the detailed minerals/metals, their concentrations, and the soil composition. According to Figure 8, the main phases of the soil were quartz, calcium carbonate, and dolomite. Also, calcium aluminum silicate and illite constituted the minor phases. The high concentrations of $\mathrm{Ca}^{+2}$ and $\mathrm{Mg}^{+2}$ detected in groundwater samples compared to their surface values, especially during the pre-monsoon period, can be explained by the soil enrichment of these cations (Table 5). Besides, XRD data confirms the presence of calcium carbonate, which forms temporary hardness as earlier presented by the results of the Piper diagrams. The result of the XRF analysis is depicted in Table 6. As shown, $\mathrm{Si}, \mathrm{Ca}$, $\mathrm{Al}, \mathrm{Fe}$, and $\mathrm{Mg}$ contributed to the highest percentage of soil composition among other identified elements, confirming their geogenic origins over anthropogenic sources. Other studies have shown that the high content of Fe in soils can 
contribute to mutagenic/carcinogenic effects on the health of the human being [61]. Because Fe and Al occur naturally at plentiful levels; thus, they hardly originated from anthropogenic inputs. Furthermore, according to Table 5, most detected heavy metals revealed the $<0.01 \%$ values in the analyzed soil, expressing no pollution. Similarly, the ICPOES data of sampled soil (Table 5) showed the abundance of $\mathrm{Ca}^{+2}, \mathrm{~K}^{+}, \mathrm{Fe}^{+2}$, and $\mathrm{Mg}^{+2}$ concentrations, which are in accordance with their high observed contents in XRF analysis. Since no heavy metal was identified as soil constituents, their presence could be attributed to anthropogenic sources such as fertilizers, sewage, and industrial discharge to the river. Although the concentration of most heavy metals was low in the sampled soil, $\mathrm{Zn}, \mathrm{Ni}$, and $\mathrm{Cr}$ amounts were noticeable, suggesting the soil of the study area is affected by anthropogenic activities. The lower detected concentration of zinc in groundwater compared to the river water samples indicate the role of the soil in the low amount of this metal and its retention in the soil matrix. An elevated zinc level has been found in groundwater in regions with mining activities, attributed to industrial waste disposal and the introduction of sludge to the environment [59].

\section{Conclusions}

In this research, various hydrogeochemical techniques and quality indices are applied to define the suitability of groundwater quality for drinking and irrigation purposes. While preliminary findings suggest that natural processes control the geochemistry of the study region, in some locations revealed anthropogenic sources were also involved. The groundwater of the study region is neutral to slightly alkaline due to the intrusion of $\mathrm{HCO}_{3}$ ions in the groundwater aquifer, as most of the samples exhibited higher levels of bicarbonate. This result is further supported by the subsurface XRD soil analysis in which calcium carbonate $\left(\mathrm{Ca}_{6} \mathrm{C}_{6} \mathrm{O}_{18}\right)$ was recognized as one of the main soil components. Hydrogeochemistry analysis reveal that the order of ions abundance were $\mathrm{Ca}^{+2}>\mathrm{Na}^{+}>\mathrm{Mg}^{+2}>\mathrm{K}^{+}$as cations and $\mathrm{HCO}_{3}^{-}>\mathrm{SO}_{4}{ }^{2-}>\mathrm{Cl}^{-}>\mathrm{NO}_{3}{ }^{-}>\mathrm{F}$-as anions. Except for $\mathrm{HCO}_{3}$ and COD, all water wells are fit with little precaution for drinking purposes, and the rest of the studied parameters are within the permissible limits set by WHO. Water quality indices and ratios for drinking and agricultural purposes demonstrated the safety of all samples for their determined uses. Contamination with a low nitrate concentration exhibits the input of anthropogenic activities like domestic sewage, animal excreta, and fertilizers application. The results from the Piper trilinear diagram demonstrated that nearly all samples belonged to $\mathrm{Ca}-\mathrm{Mg}-\mathrm{HCO}_{3}$ water type, which forms temporary hardness.

\section{Ethical Considerations}

\section{Compliance with ethical guidelines}

There were no ethical considerations to be considered in this research.

\section{Funding}

This research did not receive any grant from funding agencies in the public, commercial, or non-profit sectors.

\section{Authors' contributions}

All authors equally contributed to preparing this article.

\section{Conflict of interest}

The authors declared no conflict of interest.

\section{Acknowledgments}

The authors would like to thank for the support of the Water and Wastewater Reference Laboratory affiliated to Iran University of Medical Sciences for conducting water samples analysis and special thanks to the Ministry of Energy and Rural Water Supply Company for providing the required information.

\section{References}

[1] Choubin B, Rahmati O, Soleimani F, Alilou H, Moradi E, Alamdari N. Regional groundwater potential analysis using classification and regression trees. In: Pourghasemi HR, Gokceoglu C, editors. Spatial Modeling in GIS and R for Earth an Environmental Sciences. Amsterdam: Elsevier; 2019. pp. 485-498. [DOI:10.1016/B978-0-12-815226-3.00022-3]

[2] Adimalla N, Wu J. Groundwater quality and associated health risks in a semi-arid region of south India: Implication to sustainable groundwater management. Hum Ecol Risk Assess. 2019; 25(1-2):191-216. [DOI:10.1080/10807039.2018. 1546550]

[3] Kazi T, Arain MB, Jamali MK, Jalbani N, Afridi HI, Sarfraz RA, et al. Assessment of water quality of polluted lake using multivariate statistical techniques: A case study. Ecotoxicol Environ Saf. 2009; 72(2):301-9. [DOI:10.1016/j. ecoenv.2008.02.024] [PMID]

[4] Zhou Y, Li P, Xue L, Dong Z, Li D. Solute geochemistry and groundwater quality for drinking and irrigation purposes: A case study in Xinle City, North China. Geochemistry. 2020; 80(4):125609. [DOI:10.1016/j.chemer.2020.125609]

[5] Subramani T, Rajmohan N, Elango L. Groundwater geochemistry and identification of hydrogeochemical processes in a 
hard rock region, Southern India. Environ Monit Assess. 2010; 162(1-4):123-37. [DOI:10.1007/s10661-009-0781-4] [PMID]

[6] Matthess G. The properties of ground-water. Hoboken: Wiley; 1982. https://books.google.com/ books?id=Nt9RAAAAMAAJ\&q

[7] Jarsjö J, Andersson-Sköld Y, Fröberg M, Pietroń J, Borgström R, Löv A, et al. Projecting impacts of climate change on metal mobilization at contaminated sites: Controls by the groundwater level. Sci Total Environ. 2020; 712:135560. [DOI:10.1016/j.scitotenv.2019.135560] [PMID]

[8] Edmunds WM, Smedley PL. Residence time indicators in groundwater: The East Midlands Triassic sandstone aquifer. J Appl Geochem. 2000; 15(6):737-52. [DOI:10.1016/S08832927(99)00079-7]

[9] Thilagavathi R, Chidambaram S, Prasanna MV, Thivya C, Singaraja C. A study on groundwater geochemistry and water quality in layered aquifers system of Pondicherry region, southeast India. Appl Water Sci. 2012; 2(4):253-69. [DOI:10.1007/s13201-012-0045-2]

[10] Piper AM. A graphic procedure in the geochemical interpretation of water-analyses. Eos Trans AGU. 1944; 25(6):914-28. [DOI:10.1029/TR025i006p00914]

[11] Durov S. Natural waters and graphic representation of their composition. Dokl Akad Nauk SSSR. 1948; 59(3):87-90, https://scholar.google.com/scholar?hl=fa\&as_sdt $=0 \% 2 \mathrm{C} 5 \&$ $\mathrm{q}=$ Natural+waters+and+graphic+representation + of + their $+\mathrm{c}$ omposition\&btnG=

[12] Chadha DK. A proposed new diagram for geochemical classification of natural waters and interpretation of chemical data. Hydrogeol J. 1999; 7(5):431-9. [DOI:10.1007/ s100400050216]

[13] Kura NU, Ramli MF, Sulaiman WNA, Ibrahim S, Aris AZ. An overview of groundwater chemistry studies in Malaysia. Environ Sci Pollut Res Int. 2018; 25(8):7231-49. [DOI:10.1007/ s11356-015-5957-6] [PMID]

[14] Karmegam U, Chidambaram S, Prasanna MV, Sasidhar P, Manikandan S, Johnsonbabu G, et al. A study on the mixing proportion in groundwater samples by using Piper diagram and Phreeqc model. Chin J Geochem. 2011; 30(4):490. [DOI:10.1007/s11631-011-0533-3]

[15] Prasanna MV, Chidambaram S, Hameed AS, Srinivasamoorthy K. Hydrogeochemical analysis and evaluation of groundwater quality in the Gadilam river basin, Tamil Nadu, India. J Earth Syst Sci. 2011; 120(1):85-98. [DOI:10.1007/ s12040-011-0004-6]

[16] Uddin G, NashS, Olbert AI. A review of water quality index models and their use for assessing surface water quality. Ecol Indic. 2021; 122:107218. [DOI:10.1016/j.ecolind.2020.107218]

[17] Elsayed Gabr M, Soussa H, Fattouh E. Groundwater quality evaluation for drinking and irrigation uses in Dayrout city Upper Egypt. Ain Shams Eng J. 2021; 12(1):327-40. [DOI:10.1016/j.asej.2020.05.010]

[18] Chaudhary V, Sharma M, Yadav BS. Assessment of water fluoride toxicity levels in northwest Rajasthan, India. Fluoride. 2008; 41(3):212-5. https://scholar.google.com/ scholar?hl=fa\&as_sdt $=0 \% 2 C 5 \& q=\& b \operatorname{tnG}=$
[19] Ghassemi Dehnavi A. Hydrochemical assessment of groundwater using statistical methods and ionic ratios in Aliguodarz, Lorestan, west of Iran. J Adv Environ Health Res. 2018; 6(3):193-201. [DOI:10.22102/jaehr.2018.137767.1091]

[20] Sahu P, Sikdar P. Hydrochemical framework of the aquifer in and around East Kolkata Wetlands, West Bengal, India. Environ Geol. 2008; 55(4):823-35. [DOI:10.1007/s00254-007-1034-x]

[21] Varol S, Davraz A. Evaluation of the groundwater quality with WQI (Water Quality Index) and multivariate analysis: A case study of the Tefenni plain (Burdur/Turkey). Environ Earth Sci. 2015; 73(4):1725-44. [DOI:10.1007/s12665-014-3531-z]

[22] Kshetrimayum KS, Laishram P. Assessment of surface water and groundwater interaction using hydrogeology, hydrochemical and isotopic constituents in the Imphal river basin, Northeast India. Groundw Sustain Dev. 2020; 11:100391. [DOI:10.1016/j.gsd.2020.100391]

[23] Gaikwad SK, Kadam AK, Ramgir RR, Kashikar AS, Wagh VM, Kandekar AM, et al. Assessment of the groundwater geochemistry from a part of west coast of India using statistical methods and water quality index. HydroResearch. 2020; 3:48-60. [DOI:10.1016/j.hydres.2020.04.001]

[24] Yang J, Yu Z, Yi P, Frape SK, Gong M, Zhang Y. Evaluation of surface water and groundwater interactions in the upstream of Kui river and Yunlong lake, Xuzhou, China. J Hydrol (Amst). 2020; 583:124549. [DOI:10.1016/j.jhydrol.2020.124549]

[25] Souri L, Rabani M, Sayadi M. [Evaluation factors affecting of vulner ability the quality of groundwater and drinking wells in Pardis (Persian)]. [MSC. thesis]. Tehran: Tehran University; 2017.

[26] World Health Organization (WHO). Guidelines for drinkingwater quality. Geneva: World Health Organization; 2008. https://www.who.int/water_sanitation_health/dwq/fulltext. pdf

[27] Ramkumar T, Venkatramanan S, Anithamary I, Ibrahim SMS. Evaluation of hydrogeochemical parameters and quality assessment of the groundwater in Kottur blocks, Tiruvarur district, Tamilnadu, India. Arab J Geosci. 2013; 6(1):101-8. [DOI:10.1007/s12517-011-0327-2]

[28] Rao NS, Rao PS, Reddy GV, Nagamani M, Vidyasagar G, Satyanarayana NLVV. Chemical characteristics of groundwater and assessment of groundwater quality in Varaha River Basin, Visakhapatnam District, Andhra Pradesh, India. Environ Monit Assess. 2012; 184(8):5189-214. [DOI:10.1007/ s10661-011-2333-y] [PMID]

[29] Davis SN, De Wiest RJM. Hydrogeology. Vol. 463. New York: John Wiley \& Sons, 1966. https://www.scirp.org/ (S(czeh2tfqyw2orz553k1w0r45))/reference/ReferencesPapers.aspx?ReferenceID=1480950

[30] Ouyang Y, Nkedi-Kizza P, Wu QT, Shinde D, Huang $\mathrm{CH}$. Assessment of seasonal variations in surface water quality. Water Res. 2006; 40(20):3800-10. [DOI:10.1016/j.watres.2006.08.030] [PMID]

[31] Kim K, Koo MH, Moon SH, Yum BW, Lee KS. Hydrochemistry of groundwaters in a spa area of Korea: An implication for water quality degradation by intensive pumping. Hydrol Process. 2005; 19(2):493-505. [DOI:10.1002/hyp.5551] 
[32] Chaudhary V, Kumar M, Sharma M, Yadav BS. Fluoride, boron and nitrate toxicity in ground water of northwest Rajasthan, India. Environ Monit Assess. 2010; 161(1-4):343-8. [DOI:10.1007/ s10661-009-0750-y] [PMID]

[33] Economic and Social Commission for Western Asia. Inventory of shared water resources in western Asia. New York: United Nations; 2013. https:/ / books.google.com/books?id=QbCxgEACAAJ\&dq

[34] Marangi H, Azimzadeh AM, Nabatian GH, Kouhestani H, Mohammadi Niaei R. [Application of mineralogy and geochemistry of trace elements in determining the phases of carrying strategic elements in Angouran Zn-Pb deposit, SW Zanjan (Persian)]. Adv Appl Geol. 2017; 7(3):95-113. [DOI:10.22055/AAG.2017.13937]

[35] Veyseh S, Niazi A, GHasemi J. [Investigation of soil samples distribution in Bahabad area by ICP-MS, ICP-OES and geochemometrics analysis (Persian)]. J Geosci. 2017; 26(101):245-54. https://www.gsjournal.ir/article_41121.html?lang=en

[36] Krouse HR, Mayer B. Sulphur and oxygen isotopes in sulphate. In: Cook PG, Herczeg AL, editors. Environmental Tracers in Subsurface Hydrology. New York: Springer; 2000. pp. 195-231. [DOI:10.1007/978-1-4615-4557-6_7]

[37] Molekoa MD, Avtar R, Kumar P, Minh HVT, Kurniawan TA. Hydrogeochemical assessment of groundwater quality of Mokopane area, Limpopo, South Africa using statistical approach. Water. 2019; 11(9):1891. [DOI:10.3390/w11091891]

[38] Sidle W, Roose DL, Shanklin DR. Isotopic evidence for naturally occurring sulfate pollution of ponds in the Kankakee River Basin, Illinois-Indiana. J Environ Qual. 2000; 29(5):1594-1603. [DOI:10.2134/jeq2000.00472425002900050029x]

[39] Appelo CAJ, Postma D. Geochemistry, groundwater and pollution. $2^{\text {nd }}$ edition. Amsterdam: A.A. Balkema; 2005. https:// www.google.com/books/edition/Geochemistry_Groundwater_and_Pollution_S/F2WWnQAACAAJ?hl=en

[40] Ayenew T. The distribution and hydrogeological controls of fluoride in the groundwater of central Ethiopian rift and adjacent highlands. Environ Geol. 2008; 54(6):1313-24. [DOI:10.1007/ s00254-007-0914-4]

[41] Gomółka E, Szaynok A. [Chemia wody i powietrza (Polish)] Warsaw: Oficyna Wydawnicza Politechniki Wrocławskiej; 1997. https://fbc.pionier.net.pl/details/nnzzfsz

[42] Pulikowski K. [Zanieczyszczenia obszarowe $w$ malych zlewniach rolniczych (Polish)]. Zeszyty Naukowe Akademii Rolniczej we Wrocławiu. Rozprawy. 2004; (211):1-137. https:// agro.icm.edu.pl/agro/element/bwmeta1.element.agro-articleb90c5ca7-7925-40f4-8a20-dab63c7ada5c

[43] Orzepowski W, Kowalczyk T, Pęczkowski G, SzymańskaPulikowska A. [Zawartość wybranych składników w wodach małych zbiorników na terenach wiejskich (Polish)]. Gospodarka Wodna. 2008; (8):313-7.http://yadda.icm.edu.pl/baztech/element/bwmeta1.element.baztech-article-BPP1-0084-0066

[44] Şener Ş, Şener E, Davraz A. Evaluation of water quality using Water Quality Index (WQI) method and GIS in Aksu River (SWTurkey). Sci Total Environ. 2017; 584-584:131-44. [DOI:10.1016/j. scitotenv.2017.01.102] [PMID]

[45] Amneera WA, Najib NWAZ, Yusof SRM, Ragunathan S. Water quality index of Perlis river, Malaysia. Int J Civ Environ Eng. 2013; 13(2):1-6. https://citeseerx.ist.psu.edu/viewdoc/downloa $\mathrm{d}$ ?doi=10.1.1.654.9549\&rep=rep1\&type $=$ pdf
[46] Appelo CA, Postma D. Geochemistry, groundwater and pollution. Florida: CRC Press; 2004. [DOI:10.1201/9781439833544]

[47] Mwabi JK, Mamba BB, Momba MMB. Removal of waterborne bacteria from surface water and groundwater by cost-effective Household Water Treatment Systems (HWTS): A sustainable solution for improving water quality in rural communities of Africa. Water SA. 2013; 39(4):445-56. [DOI:10.4314/wsa.v39i4.2]

[48] Razmkhah H, Abrishamchi A, Torkian A. Evaluation of spatial and temporal variation in water quality by pattern recognition techniques: A case study on Jajrood River (Tehran, Iran). J Environ Manage. 2010; 91(4):852-60. [DOI:10.1016/j. jenvman.2009.11.001] [PMID]

[49] Rao YR, Milne JE, Marvin CH. Hydrodynamics and water quality in western Lake Ontario. J Great Lakes Res. 2012; 38(4):91-8. [DOI:10.1016/j.jglr.2012.04.001]

[50] Selvakumar S, Ramkumar K, Chandrasekar N, Magesh NS, Kaliraj S. Groundwater quality and its suitability for drinking and irrigational use in the Southern Tiruchirappalli district, Tamil Nadu, India. Appl Water Sci. 2017; 7:411-20. [DOI:10.1007/s13201-014-0256-9]

[51] Wang P, Yu J, Zhang Y, Liu C. Groundwater recharge and hydrogeochemical evolution in the Ejina Basin, northwest China. J Hydrol. 2013; 476:72-86. [DOI:10.1016/j.jhydrol.2012.10.049]

[52] Eaton FM. Significance of carbonate in irrigation water. Soil Sci. 1950; 69(2):123-34. [DOI:10.1097/00010694-19500200000004]

[53] Abdel-Satar AM, Al-Khabbas MH, Alahmad WR, Yousef WM, Alsomadi RH, Iqbal T. Quality assessment of groundwater and agricultural soil in Hail region, Saudi Arabia.

Egypt J Aquat Res. 2017; 43(1):55-64. [DOI:10.1016/j. ejar.2016.12.004]

[54] Sudhakar A, Narsimha A. Suitability and assessment of groundwater for irrigation purpose: A case study of Kushaiguda area, Ranga Reddy district, Andhra Pradesh, India. Adv Appl Sci Re. 2013; 4(6):75-81. https:/ / www.imedpub.com/abstract/suitability-and-assessment-of-groundwater-for-irrigation-purposerna-case-study-of-kushaigudaarea-ranga-reddy-district-andhra-pradesh-india-15671.html

[55] Ravikumar P, Somashekar RK. Principal component analysis and hydrochemical facies characterization to evaluate groundwater quality in Varahi river basin, Karnataka state, India. Appl Water Sci. 2017; 7(2):745-55. [DOI:10.1007/ s13201-015-0287-x]

[56] Mechal A, Birk S, Dietzel M, Leis A, Winkler G, Mogessie A, et al. Groundwater flow dynamics in the complex aquifer system of Gidabo River Basin (Ethiopian Rift): A multi-proxy approach. Hydrogeol J. 2017; 25:519-38. [DOI:10.1007/s10040016-1489-5]

[57] Katz BG, Coplen TB, Bullen TD, Davis JH. Use of chemical and isotopic tracers to characterize the interactions between ground water and surface water in mantled karst. Groundwater. 1997; 35(6):1014-28. [DOI:10.1111/j.1745-6584.1997.tb00174.x]

[58] Gibbs RJ. Mechanisms controlling world water chemistry. Science. 1970; 170(3962):1088-90. [DOI:10.1126/science.170.3962.1088] [PMID] 
[59] Li P, Wu J, Qian H, Zhang Y, Yang N, Jing L, et al. Hydrogeochemical characterization of groundwater in and around a wastewater irrigated forest in the southeastern edge of the Tengger Desert, Northwest China. Expos Health. 2016; 8(3):331-48. [DOI:10.1007/s12403-016-0193-y]

[60] Loh YSA, Akurugu BA, Manu E, Aliou AS. Assessment of groundwater quality and the main controls on its hydrochemistry in some Voltaian and basement aquifers, northern Ghana. Groundw Sustain Dev. 2020; 10:100296. [DOI:10.1016/j. gsd.2019.100296]

[61] Soodan RK, Pakade YB, Nagpal A, Katnoria JK. Analytical techniques for estimation of heavy metals in soil ecosystem: A tabulated review. Talanta. 2014; 125:405-10. [DOI:10.1016/j. talanta.2014.02.033] [PMID]

[62] Reddy VR, Reddy MS, Rout SK. Groundwater governance: A tale of three participatory models in Andhra Pradesh, India. Water Altern. 2014; 7(2):275-97. http://dlc.dlib.indiana.edu/dlc/bitstream/handle/10535/9522/plugin-file-1. pdf?sequence $=1$ 